\title{
Heegaard splittings of knot exteriors
}

\author{
YOAV MORIAH
}

\begin{abstract}
The goal of this paper is to offer a comprehensive exposition of the current knowledge about Heegaard splittings of exteriors of knots in the 3-sphere. The exposition is done with a historical perspective as to how ideas developed and by whom. Several new notions are introduced and some facts about them are proved. In particular the concept of a $1 / n$-primitive meridian. It is then proved that if a knot $K \subset S^{3}$ has a $1 / n$-primitive meridian; then $n K=K \# \cdots \# K n$-times has a Heegaard splitting of genus $n t(K)+n$ which has a 1 -primitive meridian. That is, $n K$ is $\mu$-primitive.
\end{abstract}

57M25; 57M05

\section{Introduction}

The goal of this survey paper is to sum up known results about Heegaard splittings of knot exteriors in $S^{3}$ and present them with some historical perspective. Until the mid 80's Heegaard splittings of 3-manifolds and in particular of knot exteriors were not well understood at all. Most of the interest in studying knot spaces, up until then, was directed at various knot invariants which had a distinct algebraic flavor to them. Then in 1985 the remarkable work of Vaughan Jones turned the area around and began the era of the modern knot invariants à la the Jones polynomial and its descendants and derivatives. However at the same time there were major developments in Heegaard theory of 3-manifolds in general and knot exteriors in particular. For example the invention of the notions of strongly irreducible and weakly reducible Heegaard splittings by Casson and Gordon in [8] and various techniques to deal with when two Heegaard splittings are the same by Boileau and Otal in [4]. Further work was done on the subject of distinguishing Heegaard splittings by Lustig and the author. A multitude of results were obtained, at that time, by many mathematicians, such as the Japanese school led by Kobayashi, Morimoto and Sakuma. Other results were obtained by Scharlemann, Schultens, Thompson and many others. ${ }^{1}$ It is these results that will be surveyed and discussed.

\footnotetext{
${ }^{1}$ My apologies to those I have omitted in this short list. I hope to rectify this in the body of the paper.
} 
Many of the definitions and the results which are brought here have been defined and and applied to general 3-manifolds. However they will be treated here in the more narrow context of 3-manifolds $E(K)=S^{3}-N(K)$ which are exteriors of knots in $S^{3}$. When studying Heegaard splittings of 3-manifolds there are some basic topics that we study:

Genus Determining the minimal genus of $E(K)$.

Classification This topic splits into the following:

(1) Finding inequivalent Heegaard splittings.

(2) Determining all Heegaard splittings up to equivalence (see Section 2).

(3) Deciding when Heegaard splittings are strongly irreducible or weakly reducible

(4) Understanding non-minimal genus Heegaard splittings.

Additivity Let $g(M)$ denotes the genus of the manifold $M$. Suppose $K_{1}, K_{2} \subset S^{3}$ are knots and we given $g_{1}=g\left(E\left(K_{1}\right)\right)$ and $g_{2}=g\left(E\left(K_{2}\right)\right)$. What can we say about $g\left(E\left(K_{1} \# K_{2}\right)\right)$ ?

For definitions, terminology and preliminary facts see Section 2.

In general the methods that are used to study the above topics are basically topological and algebraic. The topological methods make use of the quiver of the now standard techniques that were developed in the late eighties and early nineties, for example, the ideas of thin position introduced by Gabai to get information about Heegaard splittings of knot spaces and the application of Cerf Theory by Rubinstein and Scharlemann in order to compare two different Heegaard splittings, also the notions of strongly irreducible, weakly reducible and the Rectangle Condition introduced by Casson and Gordon. These are the technical tools that are responsible for many of the topological results. Unfortunately it is beyond the scope of this paper to describe these techniques in detail.

There are also some algebraic ideas developed by M. Lustig and the author that enable us to determine the rank of groups in many cases, in this case the fundamental group of $E(K)$. These techniques also distinguish generating systems up to Nielsen Equivalence and this was used to distinguish Heegaard splittings up to isotopy.

One of the problems that arose writing this survey was placing the many results in the different sections and sub-sections. Many of the results play a role in more than one aspect of understanding the Heegaard splittings of knot exteriors so they are mentioned not necessarily in the obvious order. 


\section{Acknowledgments}

I wish to thank the referee for numerous remarks and a meticulous job. This work was partially supported by grant 2002039 from the US-Israel Binational Science Foundation (BSF), Jerusalem, Israel.

\section{Preliminaries}

A compression body is a 3-manifold $V$ obtained from a surface $S$ cross an interval $[0,1]$ by attaching a finite number of 2-handles and 3-handles to $S \times\{0\}$. The component $S \times\{1\}$ of the boundary will be denoted by $\partial_{+} V$, and $\partial V \backslash \partial_{+} V$ will be denoted by $\partial_{-} V$. The trivial cases where $V$ is a handlebody or $V=S \times[0,1]$ are allowed.

Let $K \subset S^{3}$ be a knot. A Heegaard splitting for a knot exterior $E(K)$ is a decomposition $E(K)=V \cup_{S} W$, where $V$ is a compression body with $\partial_{+} V=S$ and $\partial_{-} V=\partial N(K)$. Two Heegaard splittings for a given $E(K)$ will be called equivalent up to homeomorphism (isotopy) if there is a homeomorphism (isotopy) $h: E(K) \rightarrow E(K)$ such that $h(S)=S$. A regular neighborhood will be denoted by $N($ ).

Historically when studying knots people used the notion of unknotting tunnels which is essentially equivalent to that of genus:

Definition 2.1 Given a knot $K \subset S^{3}$ a collection of disjoint arcs $t_{1}, \ldots, t_{n}$ properly embedded in $E(K)$ will be called an unknotting tunnel system if $E(K)-\cup\left\{N\left(t_{i}\right)\right\}$ is a handlebody. Note that $N(K) \cup\left(\cup\left\{N\left(t_{i}\right)\right\}\right)$ is always a handlebody and hence a tunnel system determines a Heegaard splitting of $S^{3}$ with the property that $K$ is contained as a core curve of one of the handlebodies.. The minimal cardinality of any such tunnel system for the given knot $K$ is called the tunnel number of $K$ and is denoted by $t(K)$.

It follows immediately that given a tunnel system with $n$ tunnels the genus, of the Heegaard splitting of $E(K)$ it determines, is $g=n+1$. In particular a minimal tunnel system determines a minimal genus Heegaard splitting of $E(K)$ and $g(E(K))=t(K)+1$.

As shall be seen it is convenient for many purposes to consider knots in $S^{3}$ as $2 n$-plats. For convenience the definition is stated below.

Definition 2.2 Given a regular projection of a braid on $2 n$ strings we can cap off consecutive pairs of strings on the top and on the bottom of the braid by small arcs, called bridges to get a projection of a knot or link in $S^{3}$. Such a projection is called a 2n-plat (see Burde and Zieschang [7]). 
Yoav Moriah

Remark 2.3 Note that:

(1) All knots and links $K \subset S^{3}$ have such projections. This follows from the theorem due to Alexander [1] that all knots and links have a representation as closed braids. There is an algorithm to obtain a braid presentation from a knot projection.

(2) If a knot $K \subset S^{3}$ has a $2 n$-plat projection then the bridge number $b(K)$ of $K$ is bounded above by $n$.

(3) A 2n-plat projection determines two sets of unknotting tunnel systems for $K$ by connecting consecutive bridges by $n-1$ small arcs (see Lustig and Moriah [37])

(4) A $2 n$-plat projection determines two Heegaard splittings of genus $n$ for $E(K)$.

(5) A $2 n$-plat projection determines two sets of $n$ generators for $\pi_{1}(E(K))$ :

Let $\hat{x}=\left\{x_{1}, \ldots, x_{n}\right\}$ be a collection of small circles around the top bridges and $\hat{y}=\left\{y_{1}, \ldots, y_{n}\right\}$ be a collection of small circles around the bottom bridges. Connect both $\hat{x}$ and $\hat{y}$ to a chosen base point. Then the curves $\hat{x}$ and $\hat{y}$ are representatives of generators.

(6) Such a projection determines two presentations for $\pi_{1}(E(K))$ :

Denote the top tunnels by $\tau_{1}, \ldots, \tau_{n-1}$ and the bottom tunnels by $\eta_{1}, \ldots, \eta_{n-1}$. Consider a small regular neighborhood of the tunnels to obtain two collections of $n-11$-handles. The cocore disks of the $\eta_{1}, \ldots, \eta_{n-1} 1$-handles determine a set of relations for the top generators and the cocore disks of the $\tau_{1}, \ldots, \tau_{n-1}$ 1-handles determine a set of relations for the bottom generators [37].

One other feature of Heegaard splittings which is relevant to this discussion is the notions of strongly irreducible and weakly reducible which are due to Casson-Gordon [8] and are defined as follows:

Definition 2.4 A Heegaard splitting $(V, W)$ of an irreducible 3-manifold $M$ will be called weakly reducible If there are essential disks $D \subset V$ and $E \subset W$ such that $\partial D \cap \partial E=\emptyset$. Otherwise $(V, W)$ will be called strongly irreducible. If there are disks such that $\partial D \cup \partial E$ is a single point $p$ we say that $(V, W)$ is stabilized.

The following is a well known definition due to Harvey which we bring for completeness:

Definition 2.5 Given a surface $S$ of genus $g \geq 2$ let $\mathcal{C}_{S}$ denote the curve complex of $S$ defined as follows:

(1) The vertex set, $\mathcal{V}_{S}$ of $\mathcal{C}_{S}$ is the set $\{[\gamma]\}$ of isotopy classes of essential simple closed curves $\gamma$ on $S$. 
(2) A set of $n$ vertices $\left\{v_{1}, \ldots, v_{n}\right\}$ represented by a set of $n$ disjoint curves will define an $(n-1)$-simplex.

Note that $\mathcal{C}_{S}$ is connected, $\operatorname{dim}\left(\mathcal{C}_{S}\right)=3 g-4$, where $g$ is the genus of $S$ and that it is not locally finite. On the 1 -skeleton of $\mathcal{C}_{S}$ there is a metric $d_{\mathcal{C}(S)}(\cdot, \cdot)$ defined by setting the length of every edge to be one.

Definition 2.6 Given a 3-manifold $M$ with a Heegaard splitting $(V, W)$, where $V$ and $W$ are compression bodies and a Heegaard surface $S$, the collection of vertices which correspond to essential curves in $S$ which bound disks in $V$ and $W$ respectively, define sub-complexes $\mathcal{D}_{V}$ and $\mathcal{D}_{W}$ of $\mathcal{C}_{S}$. They are called the disk complexes of the corresponding compression bodies.

Note that the complexes $\mathcal{D}_{V}$ and $\mathcal{D}_{W}$ of $\mathcal{C}_{S}$ are connected. The following definition is due to Hempel [18]:

Definition 2.7 Given a Heegaard splitting $(V, W)$ for a 3-manifold $M$ with $\partial_{+} V=$ $S=\partial_{+} W$, we define the distance of the Heegaard splitting, denoted by $d(V, W)$, as follows:

$$
d(V, W)=\min \left\{d_{\mathcal{C}(S)}(\partial D, \partial E) \mid D \subset V, E \subset W\right\}
$$

where $D \subset V, E \subset W$ are essential disks and $d_{\mathcal{C}(S)}(\cdot, \cdot)$ is the distance in $\mathcal{C}_{S}$. In other words, it is the distance between $\mathcal{D}_{V}$ and $\mathcal{D}_{W}$ in $\mathcal{C}_{S}$.

Note that for a given Heegaard splitting $(V, W), d(V, W)=0$ is equivalent to $(V, W)$ being reducible, $d(V, W)=1$ is equivalent to $(V, W)$ being weakly reducible but irreducible and $d(V, W) \geq 2$ is equivalent to $(V, W)$ being strongly irreducible.

Remark 2.8 Recall that all knot spaces are irreducible 3-manifolds. Hence Heegaard splittings of distance zero are stabilized.

For standard terminology and definitions see Burde and Zieschang [7], Rolfsen [63], Hempel [17] and Jaco [19]. Note also that a lot of the results apply to Heegaard splittings of 3-manifolds which are not knot spaces.

\section{Determining the genus of $E(K)$}

In theory, the problem of determining the genus for a knot space $E(K)$ is "solved" by the Rigidity Theorem proved by K Johannson in [21]: 
Theorem 3.1 (Johannson [21]) Let $M$ be a Haken 3-manifold with or without boundary and without a non-trivial, essential Stallings fibration. Then the set of all isotopy classes of Heegaard surfaces in $M$ of any given genus is finite and constructable. In particular the genus of $M$ can be determined.

Since knot spaces are Haken then for non-fibered knots we are done by the above theorem. However, in practice, for a random knot the algorithm suggested is quite impractical. Upper bounds which are not sharp are readily computable. When a knot $K \subset S^{3}$ is given as a knot projection into a plane $P$ with say, $c_{P}(K)$ crossings, the number $c_{P}(K)$ is an upper bound on its tunnel number. As, if we insert a tunnel connecting the two strings involved in each crossing we obtain a graph whose complement in $S^{3}$ is isotopic to the complement of a planar graph and hence is a handlebody. However computing the crossing number $C(K)$ of an arbitrary knot is in general very difficult. For example, computing the unknotting number for all knots with 10 or fewer crossings has only recently been accomplished by Gordon and Luecke [14] and has required work of Ozsvath-Szabo [62].

Since obtaining upper bounds which are clearly not sharp is quite easy the real question is, can we improve the upper bound? This question is on an entirely different level of difficulty for general knots.

Looking for a lower bound we note that a Heegaard splitting $(V, W)$ of $E(K)$ of genus $g$ induces a presentation :

$$
\pi_{1}(E(K))=\left\langle x_{1}, \ldots, x_{g} \mid R_{1}, \ldots, R_{g-1}\right\rangle
$$

where $\left\{x_{1}, \ldots, x_{g}\right\}$ represent generators of $\pi_{1}(W)$ given by an appropriate choice of a spine for the handlebody $W$. Thus the rank of $\pi_{1}(E(K))$ is clearly a lower bound for the genus of any Heegaard splitting.

Remark 3.2 It is still unknown if, for knots in $S^{3}$, the rank of $\pi_{1}(E(K))$ is equal to $g(E(K))$. So we do not know if the determination of $\operatorname{rank}\left(\pi_{1}(E(K))\right)$ will give a sharp lower bound for the genus. In general there are examples by Boileau and Zieschang [6] of Seifert fibered spaces $M$ for which $\operatorname{rank}\left(\pi_{1}(M)\right)=2$ but $g(M)=3$. There are results by Schultens-Weidmann [74] for graph manifolds $M$ with $\operatorname{rank}\left(\pi_{1}(M)\right)=3$ and $g(M)=4$. The question of whether rank equals genus for hyperbolic 3-manifolds is an important open question. There are some recent partial results of I Agol (unpublished), D Bachmann, D Cooper and M White [2] and last but not least J Souto [78].

For hyperbolic knots there are results regarding the rank of the fundamental group of the knot space. In [23] I Kapovitch and R Weidmann prove: 
Theorem 3.3 (Kapovitch-Weidmann [23]) If $K \subset S^{3}$ is a hyperbolic knot then there exists an algorithm which computes the rank of $\pi_{1}(E(K))$.

However, quoting Kapovitch and Weidmann themselves: "we should stress that the (above) theorem is an abstract computability result. The nature of the proof is such that it cannot provide any complexity bound on the running of the algorithm."

The moral from the above theorems is that a general algorithm to compute either the genus of the Heegaard splitting or the rank of the fundamental group will be very hard to implement. Hence we need to yield in generality in order to gain computability.

Below we present an algebraic method (devised in Lustig-Moriah [37]) for computing the rank of a general finitely generated group. It can be readily implemented to computing the rank of the fundamental group of a very large class of knots and links and the only condition that is required is that the knot/link be given in a certain $2 n$-plat projection. If a combinatorial condition, which is decided by counting, is satisfied by the $2 n$-plat, then the rank of the group is determined. The issue then becomes:

Question 3.4 Does a knot $K \subset S^{3}$ have "such" a $2 n$-plat projection? (See Lustig and Moriah [36, Theorems 0.1 and 0.4].)

A presentation, as above, determines a resolution $\varphi: F\left(X_{1}, \ldots, X_{g}\right) \rightarrow \pi_{1}(E(K))$ given by the homomorphism $\varphi: X_{i} \rightarrow x_{i}, i=1, \ldots, g$, where $F_{g}=F\left(X_{1}, \ldots, X_{g}\right)$ is the free group on $\left\{X_{1}, \ldots, X_{g}\right\}$. Let $\mathbb{Z} G$ denote the group ring of a group $G$ and $\frac{\partial}{\partial X_{i}}: \mathbb{Z} F\left(X_{1}, \ldots, X_{g}\right) \rightarrow \mathbb{Z} F\left(X_{1}, \ldots, X_{g}\right)$ be the $i$ th Fox derivative with respect to the generating system $\hat{X}=\left\{X_{1}, \ldots, X_{g}\right\}$ (for definitions and discussion of the Fox derivative see Fox [12] and Lyndon-Schupp [42]). The relations $R_{1}, \ldots, R_{g-1}$ are words in $F\left(X_{1}, \ldots, X_{g}\right)$ so we can take their Fox derivatives. Furthermore we can extend the homomorphism $\varphi$ to a ring homomorphism $\varphi: \mathbb{Z} F\left(X_{1}, \ldots, X_{g}\right) \rightarrow \mathbb{Z} \pi_{1}(E(K))$. Now let $I_{\hat{X}}$ denote the two sided ideal in $\mathbb{Z} \pi_{1}(E(K))$ generated by $\left\{\varphi\left(\frac{\partial R_{j}}{\partial X_{i}}\right)\right\}$ for $i=1, \ldots, g$ and $j=1, \ldots, g-1$, and let $\mathbb{Z} \pi_{1}(E(K)) / I_{\hat{X}}$ denote the quotient ring. Let $R$ be some commutative ring with a unit, $m \in \mathbb{N}$ and $\mathbb{M}_{m}(R)$ be the $m \times m$ matrix ring with entries in $R$.

We can now state the following theorem which is an immediate consequence of Lustig-Moriah [37, Lemma 2.1]:

Theorem 3.5 (Lustig-Moriah [37]) Suppose that for some $m \in \mathbb{N}$ and some commutative ring with a unit $R$, there is non-trivial representation $\sigma: \mathbb{Z} \pi_{1}(E(K)) / I_{\hat{X}} \rightarrow \mathbb{M}_{m}(R)$. Then $\operatorname{rank}\left(\pi_{1}(E(K))\right)=g$. 
Though at first glance implementing this theorem seems impossible due to the impenetrable nature of $\mathbb{Z} \pi_{1}(E(K)) / I_{\hat{X}}$, it turns out that in practice it is quite easy to apply. For example, for knots with a presentation for the fundamental group of $E(K)$ coming from a $2 n$-plat projection. By setting $m=1$ and $R=\mathbb{Z}\left[t, t^{-1}\right]$ there are quite a few results which were computed by hand and which will be described below.

Note that for knots $K \subset S^{3}$ in a $2 n$-plat projection we have the following inequalities:

$$
\operatorname{rank}\left(\pi_{1}(E(K))\right) \leq g(E(K)) \leq b(K) \leq n
$$

Here $b(K)$ denotes the classical bridge number of $K$ with respect to a $2-$ sphere.

Theorem 3.5 can be applied to a large class of knots and links in $S^{3}$ defined in Lustig and Moriah [36] which are called generalized Montesinos knots/links. These include in particular all 2-bridge knot/links, all Montesinos knots (for a definition see Burde and Zieschang [7, pages 196-207]) and many other much more complicated knots/links as in Lustig and Moriah [36, Theorem 5.6]. A generalized Montesinos knot/link is a combination via braids of a collection of 2-bridge knot/links each with invariants $\alpha_{i, j} / \beta_{i, j}$. If we set $\alpha=\operatorname{gcd}\left\{\alpha_{i, j}\right\}$ then we have:

Theorem 3.6 (Lustig-Moriah [36, Theorems 0.1(1) and 0.4(1)])

(1) If $\alpha \neq 1$ then $\operatorname{rank}\left(\pi_{1}(E(K))\right)=g(E(K))=b(K)=n$.

(2) If $K_{1}$ and $K_{2}$ are two generalized Montesinos knots and $K=K_{1} \# K_{2}$ and if $\alpha \neq 1$ then $t(K)=t\left(K_{1}\right)+t\left(K_{2}\right)$.

Remark 3.7 Let $K$ be a generalized Montesinos link with say $d$ components. The above results on the genus of the knot/link space $E(K)$ can be extended to closed manifolds obtained by $p_{i} / q_{i}, i=1, \ldots, d$, with $p_{i}$ even, surgery on $K$. See also [36, Theorem 5.6].

Remark 3.8 The above Theorems 3.5, 3.6 and [36, Theorem 5.6] compute the minimal genus of $E(K)$ when they can be applied. This is not a coincidence. It follows from Lustig and Moriah [37, Theorem 2.1] that there is no non-trivial representation $\sigma: \mathbb{Z} \pi_{1}(E(K)) / I_{\hat{X}} \rightarrow \mathbb{M}_{m}(R)$ if the number of generators in the presentation is not minimal.

\section{Inequivalent Heegaard splittings of knot spaces}

When trying to classify the Heegaard splittings of the exterior $E(K)$ of a given (say by a projection) knot $K \subset S^{3}$, two problems arise immediately. The first is how to 
obtain all the Heegaard splittings. The second is how to show that they are different if they happen to be of the same genus and one does not know that they are different by obvious reasons. For example, one is strongly irreducible and the other weakly reducible. (The issue of strongly irreducible and weakly reducible will be addressed in Section 5.) Heegaard splittings for $E(K)$ are equivalent to unknotting tunnel systems so the first problem can be approached by trying to find inequivalent systems of unknotting tunnels. The main tool for the second problem is the notion of Nielsen equivalence. We will discuss this first. We define:

Definition 4.1 Let $\bar{x}=\left\{x_{1}, \ldots, x_{n}\right\}$ and $\bar{y}=\left\{y_{1}, \ldots, y_{n}\right\}$ be two systems of generators for a group $G$. Denote by $F(X)$ and $F(Y)$ the free groups on bases $X=\left\{X_{1}, \ldots, X_{n}\right\}$ and $Y=\left\{Y_{1}, \ldots, Y_{n}\right\}$ respectively. Let $\beta_{x}$ and $\beta_{y}$ be the canonical epimorphisms $F(X) \rightarrow G$ given by $X_{i} \rightarrow x_{i}$ and $F(Y) \rightarrow G$ given by $Y_{i} \rightarrow y_{i}$. We will say that the generating systems $\bar{x}$ and $\bar{y}$ are Nielsen equivalent if there is an isomorphism $\alpha: F(Y) \rightarrow F(X)$ such that $\beta_{y}=\beta_{x} \circ \alpha$.

Remark 4.2 It is clear from the definition that all generating systems of cardinality $n$ of a free group of rank $n$ are Nielsen equivalent.

Suppose now that $E(K)$ has two genus $g$ Heegaard splittings $(V, W)$ and $(P, Q)$. There are spines of $W$ and $Q$ determining sets of generators $\bar{x}=\left\{x_{1}, \ldots, x_{g}\right\}$ and $\bar{y}=\left\{y_{1}, \ldots, y_{g}\right\}$ for $\pi_{1}(E(K))$. Assume that $(V, W)$ and $(P, Q)$ are isotopic. Then the isotopy takes $Q$ to $W$, say. Hence the spine of $Q$ which determines a set $\bar{y}=\left\{y_{1}, \ldots, y_{g}\right\}$ will be taken to a spine of $W$ which determines a different set $\bar{x}^{\prime}=\left\{x_{1}^{\prime}, \ldots, x_{g}^{\prime}\right\}$ of generators for $\pi_{1}(W)$. Since $\pi_{1}(W)$ is a free group the sets $\bar{x}$ and $\bar{x}^{\prime}$ are Nielsen equivalent. Hence the generators $\bar{x}$ and $\bar{y}$ are Nielsen equivalent.

If the Heegaard splittings are equivalent by a homeomorphism then the two generating sets are Nielsen equivalent up to an automorphism of $G$. (See for example Lustig and Moriah [35, 36, 37].) It follows from the above discussion that if one can show that the generating sets of $\pi_{1}(E(K))$ which are determined are Nielsen inequivalent then the corresponding Heegaard splittings are not isotopic.

\subsection{Heegaard splittings of tunnel number one knots}

Tunnel number one knots/links have genus two Heegaard splittings and hence the fundamental groups of their exteriors are generated by two elements. For these groups we have the following theorem due to Nielsen [60]: 
Theorem 4.3 (Nielsen [60]) Let $F$ be the free group with basis $\{x, y\}$ and let $w$ be the cyclic word determind by the commutator $[x, y]$. Then every automorphism of $F$ carries $w$ to $w$ or $w^{-1}$.

As an immediate consequence we have:

Theorem 4.4 Let $G$ be a two generator group. Then the commutator of the generators is, up to conjugacy and inverses, an invariant of the Nielsen class of the generating set.

Remark 4.5 Any set of words in a free group $F$ of rank greater than two which is invariant under Aut $F$ is infinite. So there is no hope for extending the above method to groups with rank bigger than two (see Lyndon and Schupp [42, Proposition 5.2]).

\subsubsection{Torus knots}

The above theorem tells us that if we can show that commutators or their inverses of the generators in two generating sets determined by two genus two Heegaard splittings are not conjugate then the Heegaard splittings are not isotopic. This idea was very successful when studying torus knots. The fundamental group of the exterior of a torus knot is in general a $\mathbb{Z}$ extension of a triangle group which embeds in $S L_{2}(\mathbb{R})$. One can find cores for the handlebodies which correspond to elliptic elements in the image of the group in $S L_{2}(\mathbb{R})$. The commutators are hyperbolic elements and hence have a translation length which is invariant under conjugation and inverses. Thus if one can show that the translation lengths of commutators corresponding to different generating sets are different the corresponding Heegaard splittings are non-isotopic. This was used to obtain the following:

Let $p, q \in \mathbb{N}$ such that $\operatorname{gcd}(p, q)=1$ and $0<p<q$. Exteriors of torus knots $T(p, q)$ are Seifert fibered spaces over the disk $D$ with two exceptional fibers $u, v$. They are denoted by $D(-r / p, s / q)$ where $r, s \in \mathbb{Z}$ such that $p s-r q=1$ and the Seifert invariants of $u$ and $v$ are $-r / p$ and $s / q$ respectively. These knots have three unknotting tunnels $\tau_{u}, \tau_{v}$ and $\tau_{m}$. The tunnel $\tau_{u}$ (resp. $\tau_{v}$ ) is obtained by connecting the fiber $u$ (resp. $v$ ) by a small arc on the base space to the boundary. The tunnel $\tau_{m}$ is a small arc connecting $u$ to $v$ on $D$. A Heegaard surface for such a Seifert fibered space will be called vertical if it is the boundary of a regular neighborhood of $u \cup \tau_{m} \cup v$ or a regular neighborhood of $\partial E(T(p, q)) \cup \tau_{u}$ or $\partial E(T(p, q)) \cup \tau_{v}$. This definition is extended to Heegaard splittings of general Seifert fibered spaces in Moriah and Schultens [50].

It was shown by Boileau-Otal in [4], and by Boileau-Rost-Zieschang in [5], that all genus two Heegaard splittings of these spaces are isotopic to the vertical ones. The 
method given by Theorem 4.4 was used in Moriah [44, 45] to distinguish the vertical Heegaard splittings and to obtain:

Theorem 4.6 (Moriah $[44,45]$ ) For a torus knot $T(p, q)$ we have:

(1) If $p \equiv \pm 1 \bmod q$ then $\tau_{m}$ is isotopic to $\tau_{u}$ and $E(T(p, q))$ has two inequivalent vertical Heegaard splittings.

(2) If $q \equiv \pm 1 \bmod p$ then $\tau_{m}$ is isotopic to $\tau_{v}$ and $E(T(p, q))$ has two inequivalent vertical Heegaard splittings.

(3) Otherwise $E(T(p, q))$ has exactly three inequivalent genus two Heegaard splittings and they are vertical.

The Heegaard splittings of torus knot exteriors of genus greater than two were dealt with in Moriah and Schultens [50]. They are stabilizations of the vertical minimal genus two splittings described above.

\subsubsection{2-bridge knots}

The study of 2-bridge knots has a long history. For an excellent exposition see Burde and Zieschang [7]. They were first classified by Schubert in [71] who showed that they are classified by a rational number $\alpha / \beta,-\alpha<\beta<\alpha$ and two knots corresponding to $\alpha / \beta$ and $\alpha^{\prime} / \beta^{\prime}$ are equivalent if and only if $\alpha=\alpha^{\prime}$ and either $\beta=\beta^{\prime}$ or $\beta \beta^{\prime} \equiv 1$ mod $\alpha$. To a continued fraction expansion of the number $\alpha / \beta$ corresponds a rational tangle so 2 -bridge knots and links can be described by a 4-plat and the top and bottom tunnels of the 4-plat give immediate candidates for genus two Heegaard splittings.

The Heegaard splittings determined by the top and bottom unknotting tunnels for 2-bridge knots which are non-amphicheiral and non-palindromic were shown to be inequivalent by Funcke [13]. He showed by a direct argument that the generating systems determined by these Heegaard splittings are Nielsen inequivalent.

This was improved upon by Bleiler-Moriah [3] for all 2-bridge knots and the distinction is up to homeomorphism. The method used was geometric, making use of the fact that tunnel number one knots are strongly invertible. Showing that the involutions are not equivalent implies that the tunnels and hence the Heegaard splittings are inequivalent as well.

Remark 4.7 In particular it is shown in [3, Theorem 5.5] that for amphicheiral 2bridge knots the Heegaard splittings determined by the top and bottom unknotting tunnels are inequivalent though the generating systems for $\pi_{1}(E(K))$ are equivalent. 
There are altogether six "more or less" obvious unknotting tunnels. These are the top and bottom tunnels $\tau_{1}, \tau_{2}$ respectively, plus two $\operatorname{arcs} \rho_{1}, \rho_{1}^{\prime}$ with end points on the left (right) top bridge and linking the right (left) top bridge once. Also two tunnels $\rho_{2}, \rho_{2}^{\prime}$ with the same construction for the bottom bridges. These unknotting tunnels were classified by Kobayashi in [27] up to homeomorphism and by Morimoto-Sakuma in [57] up to isotopy as follows:

Note that unknotting tunnels are equivalent if and only if the Heegaard splittings are equivalent. The symbol $\cong$ will denote isotopy.

Theorem 4.8 (Morimoto-Sakuma [57]) Let $K(\alpha / \beta) \subset S^{3}, \alpha / \beta \in \mathbb{Q}, \alpha$ odd, be a 2 -bridge knot. We can choose $\beta$ even. Then

(1) $\tau_{1} \cong \tau_{2}$ and $\rho_{2}, \rho_{2}^{\prime} \cong \rho_{1}$ and $\rho_{2}, \rho_{2}^{\prime} \cong \rho_{1}^{\prime}$ if and only if $\beta \equiv \pm 1 \bmod \alpha$.

(2) $\rho_{1}, \rho_{1}^{\prime} \cong \tau_{1}$ and $\rho_{2}, \rho_{2}^{\prime} \cong \tau_{2}$ if and only if $\alpha=3$.

(3) $\rho_{2}, \rho_{2}^{\prime} \cong \tau_{1}$ if and only if $\beta \equiv \pm 2 \bmod \alpha$.

(4) $\rho_{1}, \rho_{1}^{\prime} \cong \tau_{2}$ if and only if $\beta \equiv \pm 2^{-1} \bmod \alpha$.

(5) $\rho_{1}^{\prime} \cong \rho_{1}$ if and only if $\beta \equiv \pm 1 \bmod \alpha$ and $\beta \equiv \pm 2^{-1} \bmod \alpha$.

(6) $\rho_{2}^{\prime} \cong \rho_{2}$ if and only if $\beta \equiv \pm 1 \bmod \alpha$ and $\beta \equiv \pm 2 \bmod \alpha$.

Then Kobayashi, in [26], using the idea of a labeled graphic given by Cerf theory and developed by Rubinstein and Scharlemann [64] as a tool to compare Heegaard splittings showed that:

Theorem 4.9 (Kobayashi [26]) Every unknotting tunnel for a non-trivial 2-bridge knot is isotopic to one of the above six tunnels.

In a later beautiful paper Kobayashi, [29] again using the method of [64], was able to classify all the non-minimal genus Heegaard splittings of exteriors of 2-bridge knots to obtain:

Theorem 4.10 (Kobayashi [29]) Let $K$ be a non-trivial 2-bridge knot. Then, for each $g \geq 3$, every genus $g$ Heegaard splitting of the exterior $E(K)$ of $K$ is stabilized.

Now using a theorem of Hagiwara [16], stating that all genus three Heegaard splittings of $E(K)$ obtained from the six genus two ones by stabilization are isotopic, we obtain:

Theorem 4.11 (Kobayashi [29]) Let $K$ be a non-trivial 2-bridge knot. Then, for each $g \geq 3$, the genus $g$ Heegaard splittings of $E(K)$ are mutually isotopic, that is, there is exactly one isotopy class of Heegaard splittings of genus $g$.

Thus Heegaard splittings of 2-bridge knots are completely understood and are obtained by stabilizing and destabilizing one from another. 


\subsubsection{General tunnel number one knots and links}

For general tunnel number one knots there are some results given mostly in the terminology of restrictions on the knot type, assuming it has a single unknotting tunnel. We summarize below some of these results.

For genus one knots, that is, knots with a Seifert surface of genus one, Scharlemann proves the following:

Theorem 4.12 (Scharlemann [66]) Suppose $K \subset S^{3}$ has tunnel number one and genus one. Then either

(1) $K$ is a satellite knot or

(2) $K$ is a 2-bridge knot.

Tunnel number one satellite knots were classified by Morimoto-Sakuma in [57] as follows:

A knot $K \subset S^{3}$ is a $K(\alpha, \beta, p, q$,$) knot if it is constructed by gluing a non-trivial torus$ knot $K_{0}=T(p, q)$ to a 2-bridge link $L=K_{1} \cup K_{2}=K(\alpha, \beta)$, where $\alpha \geq 4$ : The component $K_{2}$ of $L$ is a trivial knot so there is a homeomorphism $h: E\left(K_{2}\right) \rightarrow N\left(K_{0}\right)$ taking a meridian of the knot $K_{2}$ to a fiber $f \subset \partial E\left(K_{0}\right)$ of the Seifert fibration of $E(T(p, q))$. The resulting knot $h\left(K_{1}\right)$ in $S^{3}$ is denoted as above.

Unknotting tunnels for $K(\alpha, \beta, p, q$,$) are obtained as follows: Let \tau_{1}$ and $\tau_{2}$ be the top and bottom tunnels for $L$. Set $a_{i}=\tau_{1} \cap \partial N\left(K_{i}\right)$ and $b_{i}=\tau_{2} \cap \partial N\left(K_{i}\right), i=1,2$. We can assume that $a_{2}$ is the end point on $\partial E(T(p, q))$ of the unknotting tunnels $\tau_{u}, \tau_{v}$ of $E\left(T(p, q)\right.$ ) (as in Section 4.1.1). For each $i \in\{1,2\}$ let $\eta_{i} \subset \partial N\left(K_{i}\right)$ be an arc joining $a_{i}$ to $b_{i}$. Set $\tau(1, u)=\tau_{1} \cup \tau_{u}, \tau(1, v)=\tau_{1} \cup \tau_{v}, \tau(2, u)=\tau_{2} \cup \eta_{2} \cup \tau_{u}$ and $\tau(2, v)=\tau_{2} \cup \eta_{2} \cup \tau_{v}$. Let $D$ denote the Dehn twist in $E(K(\alpha, \beta, p, q)$,$) along$ $\partial E(T(p, q))$ in the direction of a preferred longitude $l_{2} \in \partial E\left(K_{2}\right)$. Now choose $i \in\{1,2\}, w \in\{u, v\}$ and $n \in \mathbb{Z}$ and set $\tau(i, w, n)=D^{n}(\tau(i, w))$. We can now state:

Theorem 4.13 (Morimoto-Sakuma [57]) A tunnel number one non-simple knot in $S^{3}$ is equivalent to $K(\alpha, \beta, p, q$,$) . Any unknotting tunnel for K(\alpha, \beta, p, q$,$) is isotopic$ to $\tau(i, w, n)$ and homeomorphic to $\tau(i, w)=\tau(i, w, 0)$ for some $i \in\{1,2\}, w \in\{u, v\}$ and $n \in \mathbb{Z}$.

Remark 4.14 Note that the above theorem determines a whole infinite class of knots, that is, $K(\alpha, \beta, p, q$,$) which have finitely many minimal genus Heegaard splittings up$ to homeomorphism but infinitely many such splittings up to isotopy. 
The first such results are due to Sakuma [65] which are not published. He produces toroidal 3-manifolds obtained by gluing Seifert fibered spaces with base space a disk and two exceptional fibers $D^{2}(a / b, c / d)$ to a 2-bridge knot complement of type $K(p / q)$ so that a fiber glues to a meridian. He further requires that $a / b \neq c / d$ and that $q^{2} \neq 1 \bmod p$. These manifolds are by work of Kobayashi [25] of genus two. However one can obtain different genus two Heegaard surfaces by Dehn twisting along the gluing torus. Then using the fact that genus two Heegaard surfaces have an involution and information about the mapping class group he shows that they are non-isotopic but homeomorphic.

Remark 4.15 The generalized Waldhausen Conjecture says that a closed, orientable and atoroidal 3-manifold has only finitely many Heegaard splittings of any fixed genus, up to isotopy. This conjecture was proved by Johannson [20, 21] for Haken manifolds, which include knot exteriors. Hence the above theorem of Sakuma and Morimoto is the best possible.

We also mention some results for tunnel number one links:

An $n$-string $n \geq 1$ tangle $T$ is a pair $(B, s)$, where $B$ is a 3 -ball and $s$ is a finite collection of disjoint simple closed curves and $n$ properly embedded arcs. The tangle $T$ is essential if $\partial B-\operatorname{int}(N(s))$ is incompressible and boundary incompressible. A knot or link $L \subset S^{3}$ is an $n$-string composite if the pair $\left(S^{3}, L\right)$ has a decomposition into two essential $n$-string tangles. It will be called a tangle composite if it is an $n$-string composite for some $n$. If it is not a $n$-string composite it will be called $n$-string prime.

Theorem 4.16 (Morimoto [53]) A composite link in $S^{3}$ is tunnel number one if and only if it is a connected sum of a 2-bridge knot and the Hopf link.

Theorem 4.17 (Gordon-Reid [15]) A tunnel number one knot is $n$-string prime for all $n$.

Theorem 4.18 (Gordon-Reid [15]) A tunnel number one link in $S^{3}$ is tangle composite if and only if it has an $n$-string Hopf tangle summand for some $n$.

\subsection{Knots in $2 n$-plat projections}

Historically the first examples of inequivalent Heegaard splittings for exteriors of knots in $S^{3}$ were discovered for tunnel number one knots. In this sub-section we discuss the 
method for dealing with inequivalent Heegaard splittings of higher genus. The method is related to Theorem 3.5 above. For details see Lustig and Moriah [37].

We recall Definition 4.1: Let $\bar{x}=\left\{x_{1}, \ldots, x_{n}\right\}$ and $\bar{y}=\left\{y_{1}, \ldots, y_{n}\right\}$ be two systems of generators for a group $G$. Denote by $F(X)$ and $F(Y)$ the free groups on bases $X=\left\{X_{1}, \ldots, X_{n}\right\}$ and $Y=\left\{Y_{1}, \ldots, Y_{n}\right\}$ respectively. Let $\beta_{x}$ and $\beta_{y}$ be the canonical epimorphisms $F(X) \rightarrow G$ given by $X_{i} \rightarrow x_{i}$ and $F(Y) \rightarrow G$ given by $Y_{i} \rightarrow y_{i}$. If the generating systems $\bar{x}$ and $\bar{y}$ are Nielsen equivalent then there is an isomorphism $\alpha: F(Y) \rightarrow F(X)$ such that $\beta_{y}=\beta_{x} \circ \alpha$. This means that the words $\left\{\alpha\left(Y_{1}\right), \ldots, \alpha\left(Y_{n}\right)\right\} \in F(X)$ are a set of generators and hence are related to $X=\left\{X_{1}, \ldots, X_{n}\right\}$ by a sequence of Nielsen automorphisms. These are the collection over all $i, j \in\{1, \ldots, n\}$ of the automorphisms given by $X_{i} \rightarrow X_{i}^{-1}$ and for $i \neq j$, $X_{i} \rightarrow X_{i} X_{j}$ and their inverses. Given another system of generators $W=\left\{W_{1}, \ldots, W_{n}\right\}$ for $F(X)$, written as words in $X$, we can use the Fox derivative in $F(X)$ to obtain a "Jacobian" matrix $\left[\partial W_{i} / \partial X_{j}\right]_{i, j}$. The Jacobian matrix of a system of generators obtained by a single application of a Nielsen automorphism with respect to the original system is an elementary matrix. Recall that the Fox derivative satisfies the chain rule. Hence the matrix

$$
\left[\partial \alpha\left(Y_{i}\right) / \partial\left(X_{j}\right)\right]_{i, j}
$$

is a product of elementary matrices. The problem, of course, is that we do not know the automorphism $\alpha$. For any given $y_{i} \in \bar{y}$ the element $\alpha\left(Y_{i}\right)$ is a lift of $y_{i}$ to $F(X)$ by $\beta_{x}$. It is a theorem that any two lifts to $F(X)$ of $y_{i}$ differ by some element in the two sided ideal $I_{x} \subset \mathbb{Z} F(X)$ generated by $\left[\partial R_{i} / \partial\left(X_{j}\right)\right]_{i, j}$ where $R_{i}$ is one of the relators. Thus the matrix $\left[\beta_{x}\left(\partial \alpha\left(Y_{i}\right) / \partial\left(X_{j}\right)\right)\right]_{i, j}$ has entries in $\mathbb{Z}(G) / I_{x}$, is well defined there and is independent of $\alpha$. Furthermore it is a product of elementary matrices.

If there is a non trivial representation $\sigma: \mathbb{Z}(G) / I_{\hat{X}} \rightarrow \mathbb{M}_{m}(R)$ then, since $R$ is commutative, we can compute the determinant of the image matrix over $R$. If the two systems $\bar{x}$ and $\bar{y}$ are Nielsen equivalent then the determinant must be a unit of $R$. Showing that it is not so will prove that $\bar{x}$ and $\bar{y}$ are not Nielsen equivalent. As a consequence we have that if $\bar{x}$ and $\bar{y}$ are determined by Heegaard splitting then these Heegaard splittings are not isotopic.

Using this method for $m=1$ and $R=\mathbb{Z}\left[t, t^{-1}\right]$ the following theorems were obtained:

Theorem 4.19 (Lustig-Moriah [36]) Given a generalized Montesinos knot/link $K \subset$ $S^{3}$ in a $2 n-p l a t$ projection with associated invariants $\alpha=\operatorname{gcd}\left\{\alpha_{i, j}\right\}$ and $\beta=\prod_{i, j}\left\{\beta_{i, j}\right\}$ :

(1) If $\beta \neq \pm 1 \bmod \alpha$ then the top and bottom tunnel systems $\tau_{t}$ and $\tau_{b}$ are non-isotopic. 
(2) If $M$ is the closed 3-manifold obtained by $p_{i} / q_{i}$ surgery on the components $K_{i}$ of $K$ with $p_{i}$ even, then $\alpha \neq 1$ implies that $\operatorname{rank}\left(\pi_{1}(M)\right)=\operatorname{genus}(M)=n$. If $K$ is a knot and $\beta \neq \pm 1 \bmod \alpha$ and $2^{n-1} \neq \pm 1 \bmod \alpha$ then the Heegaard splittings of $M$ induced by $\tau_{t}$ and $\tau_{b}$ are non-isotopic.

To obtain the full strength of the theory we need to make the following definitions:

Let $L$ be the $n \times 2 n$-matrix $\left(a_{i, j}\right)$ with

$$
\left(a_{i, j}\right)= \begin{cases}1 & \text { for } j=2 i-1 \\ -1 & \text { for } j=2 i \\ 0 & \text { otherwise }\end{cases}
$$

Let $M$ be the $2 n \times n$-matrix $\left(a_{i, j}\right)$ with

$$
\left(a_{i, j}\right)= \begin{cases}1 & \text { for } i=2 j-1 \\ 1 & \text { for } i=2 j \\ 0 & \text { otherwise }\end{cases}
$$

Let $N$ be the $n \times 2 n$-matrix $\left(a_{i, j}\right)$ with

$$
\left(a_{i, j}\right)= \begin{cases}1 & \text { for } j=2 i-1 \\ 0 & \text { otherwise }\end{cases}
$$

With these conventions we have the following theorem for a very general link $K \subset S^{3}$ with a $2 n$-plat projection:

Theorem 4.20 (Lustig-Moriah [36]) Let $B$ denote the $2 n$-braid underlying the $2 n$-plat projection of $K$. Consider the image $\hat{\rho}(B)$ of $B$ under the Burau representation of the $2 n$-braid group, with the variable $t$ evaluated at -1 . Let $\alpha$ be the greatest common divisor of the entries of the matrix $L \circ \hat{\rho}(B) \circ M$. Let $\beta$ be the determinant of the matrix $N \circ \hat{\rho}(B) \circ M$. Then

(1) If $\alpha \neq 1$ then $\operatorname{rank}\left(\pi_{1}(E(K))\right)=g(E(K))=b(K)=n$.

(2) If $K_{1}$ and $K_{2}$ are two knots, $K=K_{1} \# K_{2}$ and $\alpha \neq 1$ then $t(K)=t\left(K_{1}\right)+t\left(K_{2}\right)$.

(3) If $\alpha \neq 1$ and $\beta \neq \pm 1 \bmod \alpha$ then the top and bottom tunnel systems $\tau_{t}$ and $\tau_{b}$ for $K$ are non-isotopic.

(4) If $M$ is the closed 3-manifold obtained by $p_{i} / q_{i}$ surgery on the components $K_{i}$ of $K$ with $p_{i}$ even, then $\alpha \neq 1$ implies that $\operatorname{rank}\left(\pi_{1}(M)\right)=\operatorname{genus}(M)=n$. If $K$ is a knot and $\beta \neq \pm 1 \bmod \alpha$ and $2^{n-1} \neq \pm 1 \bmod \alpha$ then the Heegaard splittings of $M$ induced by $\tau_{t}$ and $\tau_{b}$ are non-isotopic. 
That is, the statements of Theorem 3.6 and statements (1) and (2) of Theorem 4.19 hold for $K, \alpha$ and $\beta$.

Remark 4.21 It should be emphasized that the class of knot/links which are described by the condition of Theorem 4.20 is much larger than that of generalized Montesinos knots/links.

We end this section with a result obtained by the method discussed above to distinguish minimal genus Heegaard splittings. It shows that the Heegaard structure of minimal genus Heegaard splittings for knot exterior spaces is indeed a rich one.

Theorem 4.22 (Lustig-Moriah [38]) For each $g \geq 3$ there are infinitely many hyperbolic 3-manifolds of genus $g$ with at least $2^{g}-2$ pairwise non-homeomorphic minimal genus Heegaard splittings. These are obtained by $m / n$ surgery, $m$ even, on a Montesinos knot $K=\mathbf{m}\left(e ;\left(\alpha_{1}, \beta_{1}\right), \ldots,\left(\alpha_{g}, \beta_{g}\right)\right)$ where the $\beta_{i}$ 's are mutually distinct odd primes and $\alpha=\operatorname{gcd}\left(\alpha_{1}, \ldots, \alpha_{g}\right)>2^{2 g-1}\left(\beta_{1} \cdots \beta_{g}\right)^{2}$. The exterior $E(K)$ has at least $2^{g}-1$ mutually non-homeomorphic unknotting tunnel systems, that is, $2^{g}-1$ mutually non-homeomorphic minimal genus Heegaard splittings.

\section{Strongly irreducible and weakly reducible}

As all knot exteriors are irreducible, a given Heegaard splitting for a knot space is either stabilized or if not it is either strongly irreducible or weakly reducible as in Definition 2.4. So the question of determining the nature of a given Heegaard splitting is split into three:

(1) How do we show that a Heegaard splitting for $E(K)$ is stabilized?

(2) How do we show that a Heegaard splitting of $E(K)$ is strongly irreducible?

(3) How do we show that a Heegaard splitting of $E(K)$ is weakly reducible but not stabilized?

Question (1) was discussed in Section 3. If the Heegaard splitting is of minimal genus then it is clearly non-stabilized. If it is not minimal genus then the known algebraic techniques at our disposal fail and in some special cases, that is, exteriors of 2-bridge knot some things can be said (see Theorem 4.10 and Kobayashi [29]).

If the Heegaard splitting is strongly irreducible then it is clearly non-stabilized and then the distinction between minimal and non-minimal genus is not relevant to answering Question (2) above. Below we will describe some results regarding Question (2). 
If it is weakly reducible then it could be stabilized or not depending on whether the distance of the Heegaard splitting, as in Definition 2.7, is zero or one. If the Heegaard splitting is of minimal genus then the fact that it is non-stabilized is obvious and below we will show examples of such Heegaard splittings. We are left with weakly reducible non minimal genus Heegaard splittings. It turns out that to decide whether a Heegaard splitting is distance one if it is not minimal is a very hard question indeed. In fact the following remark should be emphasized:

Remark 5.1 There is no known example of a non-minimal genus distance one Heegaard splitting of a 3-manifold which is either closed or with a single boundary component ${ }^{2}$.

\subsection{Strongly irreducible Heegaard splittings of knot spaces}

We begin with a series of definitions leading to the definition of the Rectangle Condition due to Casson and Gordon.

Definition 5.2 Let $S$ be a genus $g$ orientable surface and $\mathcal{C}=\left\{c_{1}, \ldots, c_{k}\right\}$ a collection of disjoint essential simple closed curves on $S$. A wave with respect to $\mathcal{C}$ is an $\operatorname{arc} \omega$ so that:

(1) $\partial \omega=\left\{p_{1}, p_{2}\right\}$ is contained in a single component $c_{j_{0}} \in \mathcal{C}$.

(2) $\omega$ meets $c_{j_{0}}$ from the same side.

(3) $\omega$ is not homotopic rel. $\partial \omega$ into $c_{j_{0}}$.

Let $V$ be a handlebody/compression body and let $\mathcal{C}=\left\{c_{1}, \ldots, c_{k}\right\}$ be a maximal collection of disjoint non-isotopic essential simple closed curves on $\partial V$ which bound disks $\mathcal{D}=\left\{d_{1}, \ldots, d_{k}\right\} \subset V$ (that is, $\mathcal{D}$ is a simplex of maximal dimension in $\mathcal{D}_{V}$ as in Definition 2.6). The boundary $\partial D$ of any essential disk $D \subset V$ is either parallel to one of the disks in $\mathcal{D}$ or has at least two waves with respect to $\mathcal{C}$. Such a collection $\mathcal{D}$ will be called a pair of pants decomposition for $V$. A 3-ball component of $V-\mathcal{D}$ will be called a solid pair of pants.

Definition 5.3 (Rectangle Condition) A Heegaard splitting $(V, W)$ for a 3-manifold $M$ will satisfy the Rectangle Condition if there is a blue pants decomposition $\mathcal{D}$ for $V$ and a a red pants decomposition $\mathcal{E}$ for $W$ so that every pair of blue curves in every solid pair of pants of $\partial \mathcal{D}$ meets every pair of red curves of every solid pair of pants in $\partial \mathcal{E}$.

${ }^{2}$ For further discussion of this issue see discussion at the end of Section 6. 
Remark 5.4 If $V$ is a compression body with $\partial_{-} V=T^{2}$ we assume that a maximal collection of disks in $V$ will include a separating disk $D_{0}$ that cuts off a $T^{2} \times I$ component from $V$. In this case a maximal collection $\mathcal{D}$ will contain $3 g-4$ disks. Note that no essential disk in $V$ can have a wave in $T^{2} \times I$.

Remark 5.5 Note that a Heegaard splitting which satisfies the Rectangle Condition is strongly irreducible: Any disk in $V(W)$ is either parallel into one of the curves $d_{i}\left(e_{j}\right)$ or has a wave with respect to $\mathcal{D}(\mathcal{E})$. A wave travels parallel to the two disks in the pair of pants which contains it but do not contain the wave's endpoints. Hence the Rectangle Condition implies that any two essential disks intersect. In fact they intersect in waves.

Note also that the Rectangle Condition is strictly stronger than that of strongly irreducible: The standard Type 2 splittings of (surface) $\times I$, are strongly irreducible but have pairs of essential disks that intersect exactly twice. However Sedgwick proves in [76]:

Lemma 5.6 Let $V \cup_{S} W$ be a Heegaard splitting. Let $D \subset V, E \subset W$ be essential disks. If there are complete collections of disks $\Delta \subset V, \Delta^{\prime} \subset W$ which satisfy the Rectangle Condition then $|\partial D \cap \partial E| \geq 4$.

Remark 5.7 It should also be pointed out that there are stronger notions then the Rectangle Condition. This is the Double Rectangle Condition of Lustig-Moriah see [41].

Remark 5.8 Given a Heegaard splitting for a 3-manifold or a knot space with a pants decomposition, the question of, does it satisfy the rectangle condition, becomes a combinatorial one. So one can view having the Rectangle Condition property as a combinatorial approximation to that of being strongly irreducible.

Remark 5.9 Casson and Gordon who defined the Rectangle Condition in unpublished work (see Moriah and Schultens [50]) used it to show that there are closed 3-manifolds which have strongly irreducible Heegaard splittings. A detailed description of these examples can be found in work of Sedgwick [75].

Theorem 5.10 (Casson-Gordon) Let $K=K\left(p_{1}, \ldots, p_{r}\right)$ be a pretzel knot in $S^{3}$ with $r>5$ strands, $p_{i}>2$ and each $p_{i}$ odd. Let $M$ be a manifold which is obtain by $\frac{1}{k}$-surgery for $k \geq 6$. Then the Heegaard splitting of $M$ obtained from a regular neighborhood of a Seifert surface for $K$ and its complement is strongly irreducible. 
In [40] M Lustig and the author also used the Rectangle Condition to show that in fact the phenomenon of manifolds with strongly irreducible Heegaard splittings is quite common and that there are many knots in $S^{3}$ which produce after surgery such 3-manifolds. Kobayashi in [27] also exhibits manifolds with strongly irreducible Heegaard splittings. These examples will be further discussed in Section 6, Section 6.1. Recently Minsky, Moriah and Schleimer have showed in [43] that for any positive integer $t \geq 1$ and $n>0$ there are tunnel number $t$ knots $K \subset S^{3}$ so that $E(K)$ has a Heegaard splitting of distance greater than $n$. In particular they are all strongly irreducible and in fact it seems knots with high distance Heegaard splittings are the "generic" case.

Many of the definitions and results which come under the above "Strongly irreducible Heegaard splittings of knot spaces" heading of this section are also discussed in depth in Sections 6.1 and 7.1. We refer the reader to those sections.

\subsection{Weakly reducible non-stabilized Heegaard splittings}

The previous section tells us that knots in $S^{3}$ with strongly irreducible Heegaard splittings are abundant. So where does one look for knots with weakly reducible Heegaard splittings? The first such result is due to M Lustig and the author [39]. In that paper the notion of a wide knot is defined as follows:

Definition 5.11 (a) A $2 n$-braid will be called wide if in its standard projection where every crossing is replaced by a node, there is no monotonically descending path connecting the top of the second strand to the bottom of the $(2 n-1)$-strand or vice versa.

(b) A 2n-plat projection of a knot or link will be called wide if the underlying braid is wide.

(c) A knot or link $K \subset S^{3}$ will be called wide if it has a wide $2 n$-plat projection so that the corresponding Heegaard splitting is irreducible.

Then the following proposition is proved:

Proposition 5.12 (Lustig-Moriah [39]) For every knot or link $K$ in $S^{3}$ in a wide $2 n$-plat projection both canonical Heegaard splittings are weakly reducible. In particular every wide knot or link has a weakly reducible and irreducible (distance one) Heegaard splitting. 
Idea of Proof Consider the canonical Heegaard splitting of $E(K)$ induced by inserting the bottom tunnels to the $2 n$-plat projection. Embed a 2 -sphere $S$ in $\left(S^{3}, K\right)$ which cuts off the "top" bridges. When a regular neighborhood $N(K)$ is removed from $S^{3}$ to obtain $E(K)$ the "inside" of $S$ (that is, the 3-ball (before removing $N(K)$ ) containing the top bridges) is isotopic to $W$ of the Heegaard splitting. It is clear that the leftmost bridge defines a dual essential disk $D$ in $W$. Since the knot/link is wide then in the process of gluing the handlebody to the compression body the boundary of the disk $D$ will be mapped according to the braid. Hence it will not meet the cocore disk of the rightmost bottom tunnel which is an essential disk in the compression body component $V$ of the Heegaard splitting.

The fact that the Heegaard splitting is irreducible is proved for many knots/links in a $2 n$-plat projection using the algebraic techniques of Theorem 3.6.

Another place to look for knots which have Heegaard splittings of distance one are knots which are connected sums. This approach was taken by the author in [47]. We first need some definitions.

Definition 5.13 We say that a curve on a handlebody is primitive if there is an essential disk in the handlebody intersecting the curve in a single point. An annulus $A$ on the boundary of a handlebody is primitive if its core curve is a primitive curve. A Heegaard splitting $(V, W)$ for $E(K)$, where $\partial E(K) \subset V$ will be called $\gamma$-primitive if there is a spanning annulus $A \subset V$ such that $\partial A=\gamma \cup \alpha$ where $\gamma \subset \partial E(K)$ and $\alpha$ is a primitive curve on the Heegaard surface $\partial W$. If $\gamma$ is a meridian for $K$ then we say that $(V, W)$ is $\mu$-primitive.

We say that a knot $K \subset S^{3}$ has a primitive meridian, or is $\mu$-primitive if $E(K)$ has a minimal genus Heegaard splitting with a primitive meridian.

Definition 5.14 Two Heegaard splittings $\left(V_{i}, W_{i}\right)$ for $E\left(K_{i}\right)$ respectively, induce a decomposition of $E(K)=E\left(K_{1} \# K_{2}\right)$ into $(V, W)$. We can think of $V_{i}$ as a union of $\partial E\left(K_{i}\right) \times I$ and 1-handles. Let $t_{i} \subset K_{i}$ be an arc. Let $B_{i}$, a small 3-ball in $S^{3}$ such that $t_{i}$ is an unknotted properly embedded $\operatorname{arc}$ in $B_{i}$, the closure of $B_{i} \cap S^{3}-E\left(K_{i}\right)$ is a regular neighborhood $N\left(t_{i}\right)$ of $t_{i}$ in $B_{i}$ and the annulus $A_{i}^{\prime}=\partial B_{i}-N\left(\partial t_{i}\right)$ is the union of two vertical annuli $A_{1}^{* i}, A_{2}^{* i}$ and a meridional annulus $A_{i} \subset \partial E\left(K_{i}\right) \times\{1\} \subset \partial_{+} V_{i}=\partial W_{i}$. We regard $\left(B_{i}, t_{i}\right)$ as the pair which is removed from $\left(S^{3}, K_{i}\right)$ when forming the connected sum of pairs $\left(S^{3}, K_{1} \# K_{2}\right)=\left(S^{3}, K_{1}\right) \#\left(S^{3}, K_{2}\right)$. We obtain $V$ as follows:

Cut the compression bodies $V_{1}$ and $V_{2}$ open along the annuli $A_{1}^{* 1}, A_{2}^{* 1}$ and $A_{1}^{* 2}, A_{2}^{* 2}$ respectively. Consider the components which are not $B_{i}-N\left(t_{i}\right)$. We obtain handlebodies $V_{1}^{\prime}$ and $V_{2}^{\prime}$ with copies of $A_{1}^{* 1}, A_{2}^{* 1} \subset V_{1}^{\prime}$ and copies of $A_{1}^{* 2}, A_{2}^{* 2} \subset V_{2}^{\prime}$. Now glue $V_{1}^{\prime}$ to 
$V_{2}^{\prime}$ by identifying $A_{1}^{* 1}$ with $A_{1}^{* 2}$ and $A_{2}^{* 1}$ with $A_{2}^{* 2}$ to get a compression body $V$. We obtain $W$ by gluing $W_{1}$ and $W_{2}$ along the meridional annulus $A_{i}$. Hence $V$ is always a compression body but $W$ is a handlebody if and only if the meridional annulus $A_{i}$ is a primitive annulus in $\partial W_{i}$ for one of $i=1$ or $i=2$. In this case we will say that $(V, W)$ is the induced Heegaard splitting of $E(K)$ induced by $\left(V_{1}, W_{1}\right)$ and $\left(V_{2}, W_{2}\right)$.

Theorem 5.15 (Moriah [47]) Let $K_{1}, K_{2}$ and $K=K_{1} \# K_{2}$ be knots in $S^{3}$ and $\left(V_{i}, W_{i}\right), i=1,2$ be Heegaard splittings for $E\left(K_{i}\right)$. If $\left(V_{1}, W_{1}\right)$ and $\left(V_{2}, W_{2}\right)$ induce a Heegaard splitting $(V, W)$ of $E(K)$ then $(V, W)$ is a weakly reducible Heegaard splitting.

Using the above definitions we have the following two theorems:

Theorem 5.16 (Moriah [47]) Given knots $K_{1}, K_{2}$ and $K=K_{1} \# K_{2}$ in $S^{3}$ for which the tunnel number satisfies $t(K)=t\left(K_{1}\right)+t\left(K_{2}\right)+1$ then there is an induced minimal genus Heegaard splitting of $E(K)$ which is weakly reducible.

These theorems raise the following two questions:

Question 5.17 Are there non-prime knots in $S^{3}$ so their exteriors have strongly irreducible minimal genus Heegaard splittings?

Question 5.18 Are there knots in $S^{3}$ so their exteriors have both weakly reducible and strongly irreducible minimal genus Heegaard splittings?

Remark 5.19 There is a positive ansewer to Question 5.17. The first example was given by the author in [47]. There $K_{1}=K^{n}(-2,3,-3,2)$ is the twisted pretzel where between the two 3-strands of twists one introduces an odd number $n \in \mathbb{Z}, n \notin\{-1,0,1\}$ of "horizontal" crossings, and $K_{2}$ is any 2 -bridge knot. Then $t\left(K_{1}\right)=2$ and $t\left(K_{2}\right)=1$. It has been used by Morimoto [54] to show that the tunnel number can go down after connected sum, that is, it is sub-additive. He exhibits a Heegaard splitting of genus three so that $t\left(K_{1} \# K_{2}\right)=2<2+1=t\left(K_{1}\right)+t\left(K_{2}\right)$ (see Section 7, Section 7.3). We have:

Proposition 5.20 (Moriah [47]) Let $K_{1}$ and $K_{2}$ be as above. The genus three Heegaard splitting of $E\left(K_{1} \# K_{2}\right)$ described in [54] is strongly irreducible.

This result was generalized by Kobayashi and Rieck in [32]. They prove:

Theorem 5.21 (Kobayashi and Rieck [32, Corollary 5.4]) Let $K_{1}$ and $K_{2}$ be knots in closed orientable 3-manifolds. Let $X_{i}=E\left(K_{i}\right)(i=1,2)$ and $X=E\left(K_{1} \# K_{2}\right)$. Suppose that: 
(1) $X$ is irreducible,

(2) $X_{i},(i=1,2)$ does not contain a meridional essential annulus,

(3) $X_{i},(i=1,2)$ does not contain an essential torus, and

(4) $g\left(X_{1}\right)+g\left(X_{2}\right) \geq 5$ and $g(X)=3$.

Then any minimal genus Heegaard splitting of $X$ is strongly irreducible.

Question 5.18 is still open for manifolds which are exterior of knots in $S^{3}$. However for general 3-manifolds it has been answered positively by Kobayashi and Rieck. They have proved:

Theorem 5.22 (Kobayashi and Rieck [32]) There are infinitely many 3-manifolds which have both strongly irreducible and weakly reducible Heegaard splittings of minimal genus.

Idea of proof The manifolds $M$ are constructed as follows: Let $X=S^{3}-N\left(K_{1}\right)$, $Y=S^{3}-N\left(K_{2}\right)$ and $Z=S^{3}-N\left(K_{3}\right)$, where $K_{1}=T(2,3)$ is the trefoil knot, $K_{2}=L(\alpha, \beta)$ with $\alpha$ even, is any 2 -bridge link which is not the Hopf link and $K_{3}=K(2,5)$ is the figure 8 knot. Let $\mu_{1}$ and $\mu_{2}$ be meridians of the 2 -bridge link $L(\alpha, \beta)$ components, $\lambda$ be the longitude of $K_{3}$ and $\gamma$ be the boundary of the cabling annulus in $X$.

Attach $X$ and $Z$ to $Y$ by gluing their tori boundaries so that $\gamma$ is mapped to $\mu_{1}$ and $\lambda$ to $\mu_{2}$. We obtain a closed 3-manifold $M$. By analyzing the incompressible tori contained in $M$ they conclude that $g(M)=3$ and that $M$ has a strongly irreducible minimal genus Heegaard splitting. On the other hand $M$ has a Heegaard splitting which is the amalgamation of two genus two Heegaard splittings along a torus. Hence it is of genus three and is clearly weakly reducible.

\section{Non-minmal genus Heegaard splittings}

In general non-minimal genus Heegaard splittings of exteriors of knots in $S^{3}$ are not well understood at all. The only comprehensive results are those of Kobayashi and Moriah-Schultens mentioned in Section 4.1, Subsections 4.1.2 and 4.1.1. That is Theorem 4.10 and Theorem 4.11, where all Heegaard splittings of $E(K)$ for $K$ a 2 -bridge knot are classified. Also complements of torus knots are Seifert fibered spaces and as such Moriah-Schultens [50, Theorem 0.1] states that any of their non-minimal Heegaard splittings is a stabilization of minimal genus two vertical Heegaard splittings. 
For manifolds with non-empty boundary there is one other technique for obtaining non-minimal genus Heegaard splittings other than stabilizing. It is called "boundary stabilization", defined by the author in [46]. Boundary stabilization uses an operation on Heegaard splittings called "amalgamation". Amalgamated Heegaard splittings were defined by Schultens [72]. We give the full definition for the benefit of the reader:

Definition 6.1 Given two manifolds $M_{1}$ and $M_{2}$ with respective Heegaard splittings $\left(U_{1}^{1}, U_{1}^{2}\right)$ and $\left(U_{2}^{1}, U_{2}^{2}\right)$, assume further that there are homeomorphic boundary components $F_{1} \subset \partial_{-} U_{1}^{1}$ and $F_{2} \subset \partial_{-} U_{2}^{1}$. Denote the homeomorphism $F_{1} \rightarrow F_{2}$ by $g$. Let $M$ be a manifold obtained by gluing $F_{1}$ and $F_{2}$ along the homeomorphism $g$. We can obtain a Heegaard splitting $\left(V_{1}, V_{2}\right)$ for $M$ by a process called amalgamation as follows:

Given a compression body $U$ we can assume that it has the structure of $\partial_{-} U \times$ $I \cup(\cup\{1$-handles $\})$. Let $h_{i}$ be the homeomorphism $N\left(\partial_{-} U_{i}^{1}\right) \rightarrow\left(\partial_{-} U_{i}^{1}\right) \times I$ and $p_{i}:\left(\partial_{-} U_{i}^{1}\right) \times I \rightarrow \partial_{-} U_{i}^{1}$ the projection into the first factor. Define an equivalence relation $\equiv$ on $M_{1}, M_{2}$ as follows:

(1) If $x_{i}, y_{i} \in N\left(F_{i}\right)$ are points such that $p_{i} h_{i}\left(x_{i}\right)=p_{i} h_{i}\left(y_{i}\right)$ then $x_{i} \equiv y_{i}$.

(2) If $x \in F_{1}, y \in F_{2}$ and $g(x)=y$, where $g: F_{1} \rightarrow F_{2}$ is the homeomorphism between the surfaces, then $x \equiv y$.

Furthermore we can arrange that the attaching disks on $F_{1} \times\{1\},\left(F_{2} \times\{1\}\right)$ for the 1-handles in $U_{1}^{1}, U_{2}^{1}$ respectively, have disjoint images in $F_{1}\left(F_{2}\right)$ and hence they do not get identified to each other. Now set $M=\left(M_{1} \cup M_{2}\right) / \equiv, V_{1}=\left(U_{1}^{1} \cup U_{2}^{2}\right) / \equiv$, $V_{2}=\left(U_{1}^{2} \cup U_{2}^{1}\right) / \equiv$. Note that $V_{1}=U_{2}^{2} \cup N\left(F_{1}\right) \cup(\cup\{1$-handles $\}) \cup\left(\partial_{-} U_{1}^{1}-F_{1}\right) \times I$ and $V_{2}=U_{1}^{2} \cup N\left(F_{2}\right) \cup(\cup\{1$-handles $\}) \cup\left(\partial_{-} U_{2}^{1}-F_{1}\right) \times I$ so that $V_{1}$ and $V_{2}$ are compression bodies defining a Heegaard splitting $\left(V_{1}, V_{2}\right)$ for $M$. The Heegaard splitting $\left(V_{1}, V_{2}\right)$ of $M$ is called the amalgamation of the Heegaard splittings $\left(U_{1}^{1}, U_{1}^{2}\right)$ of $M_{1}$ and $\left(U_{2}^{1}, U_{2}^{2}\right)$ of $M_{2}$ along $F_{1}$ and $F_{2}$.

We give here a modified version for knot spaces:

Definition 6.2 Given a genus $g$ Heegaard splitting for a knot exterior $E(K)$ in $S^{3}$ we can obtain a genus $g+1$ Heegaard splitting by taking the standard genus two Heegaard splitting for $S^{1} \times S^{1} \times I$ (see Scharlemann and Thompson [69]) and amalgamating the two splittings via the identity map $T^{2} \times\{1\} \rightarrow \partial E(K)$. The amalgamated Heegaard splitting will be called a boundary stabilization. 
It is clear that if the knot is $\gamma$-primitive (see Definition 5.13 in Section 5) then a boundary stabilized Heegaard splitting is in fact stabilized: Since the knot is $\gamma$-primitive then after gluing $T^{2} \times I$ to $\partial E(K)$ there is an annulus $A$ in $T^{2} \times I \cup E(K)$ which meets a disk $D$ in the handlebody component of the non-stabilized Heegaard splitting in a single point. It is just the extension of the annulus in $E(K)$ which has the same property. The amalgamation process removes a neighborhood of an essential arc from $A$ thus creating an essential disk $E$ in the handlebody component of the boundary stabilized Heegaard splitting. The disk $E$ still meets the disk $D$ (now in the compression body component of the boundary stabilized Heegaard splitting) in a single point (see [46]). So the boundary stabilized Heegaard splitting is stabilized. However if the knot is not $\mu$ or $\gamma$-primitive then the following conjecture is proposed:

Conjecture 6.3 Given a knot $K \subset S^{3}$ which is not $\gamma$-primitive then a boundary stabilization of a minimal genus Heegaard splitting of $E(K)$ is non-stabilized.

\subsection{Manifolds with arbitrarily high genus irreducible Heegaard splittings}

Though this sub-section discusses closed manifolds, knot exteriors played a crucial role in showing that there are 3-manifolds with irreducible Heegaard splittings of arbitrarily high genus.

The actual result of Casson and Gordon discussed in Section 5.1, Remark 5.9, is much stronger than the one stated. They used the Rectangle Condition to show that there are closed 3-manifolds which have strongly irreducible Heegaard splittings of, in fact, arbitrarily high genus. This result is unpublished and a proof of it, similar to the original proof, is given by Kobayashi [24]. A different proof due to Casson is given in the appendix of Moriah-Schultens [50]. These manifolds are obtained by $\frac{1}{k}$-surgery for $k \in \mathbb{Z}, k \geq 6$ on pretzel knots in $S^{3}$ of the form $K\left(p_{1}, \ldots, p_{r}\right)$. These are pretzel knots with $r>5$ strands and each strand has $p_{i}>2$ and odd crossings.

Results of M Lustig and the author [40] showed that in fact this phenomenon is quite common and that there are many knots in $S^{3}$ which produce after surgery 3-manifolds with strongly irreducible Heegaard splittings of arbitrarily high genus. These knots are given as knots which embed on the boundary of regular neighborhoods of planar graphs $N(\Gamma)$ called trellises. Trellises generalize the notion of a $2 n-$ plat and allow us to present a knot or link carried by them by a family of integer parameters, assembled in a twist matrix. We can compute a trellis linking number $a(K)$. It is clear that both $N(\Gamma)$ and $S^{3}-N(\Gamma)$ are handlebodies. Then one obtains a closed manifold by doing a $(1+k a(K)) / k$-surgery on $E(K)$, for any $k \in \mathbb{Z}$. This surgery is so designed to actually 
correspond to a Dehn twist on $\partial N(\Gamma)$. A Heegaard splitting that is created in this way is called horizontal. Once the two handlebodies are glued together with this Dehn twist we get the required closed manifold. The knots satisfy some complexity to ensure that the Rectangle Condition for these Heegaard splittings will be satisfied so that we get a strongly irreducible Heegaard splitting. We then have:

Theorem 6.4 Let $K$ be a knot given as a $2 n$-plat in $S^{3}$, and assume that all twist coefficients of the underlying braid satisfy $\left|a_{i, j}\right| \geq 3$. Then for all $k \in \mathbb{Z}$, with $|k| \geq 6$, the manifold $K((1+k a(K)) / k)$ has a strongly irreducible horizontal Heegaard splitting.

There are planar graphs $T$ which are a generalization of trellisses. If we consider generalized trellisses $T$ with a particular combinatorial feature, called an interior pair of edges, we can perform flypes on these more general knots in a way similar to that done by Casson-Gordon in [50] for pretzel knots. Define the "genus" of $T$ by $g(T)=-2(\chi(T))$.

Theorem 6.5 Let $T$ be a generalized trellis and let $K=K(A) \subset S^{3}$ be a knot carried by $T$ with twist matrix $A$. Assume that all coefficients $a_{i, j}$ of $A$ satisfy $\left|a_{i, j}\right| \geq 3$ and that there is an interior pair of edges $\left(e_{i, j}, e_{i, h}\right)$ of $T$ with twist coefficients $\left|a_{i, j}\right|,\left|a_{i, h}\right| \geq 4$. Then for all $k, n \in \mathbb{Z}$, with $|k| \geq 6$, the manifolds $K((1+k a(K)) / k)$ have irreducible Heegaard splittings $\Sigma(n)$ of arbitrarily large genus $g(T)+2 n$, all of which are horizontal.

Remark 6.6 In particular, for $K$ as in Theorem 6.5, all of the splittings $\Sigma(n)$, stabilized once, are stabilizations of a common low genus Heegaard splitting.

There are other examples of manifolds with strongly irreducible Heegaard splittings of arbitrarily high genus. These manifolds were introduced by Kobayashi in [27]. Kobayashi considers two component links $L^{n}=l_{1}^{n} \cup l_{2}^{n} \subset S^{3}$ which are pretzels of the form

$$
P(9,-9,7,5,-5-7,5,5,-5,-5, \ldots, 5,5,-5,-5) \text {, }
$$

where there are $n-1$ tangles of the form $(5,5,-5,-5)$. Two Seifert surfaces $S, S^{\prime}$ in $E(L)$ will be called weakly equivalent if there is a homeomorphism $h: E(L) \rightarrow E(L)$ so that $h(S)=S^{\prime}$. For these links we have:

Proposition 6.7 (Kobayashi [27]) For each integer $g \geq n$, the link $L^{n}$ has $\left(\begin{array}{l}g-1 \\ n-1\end{array}\right)$ free, incompressible Seifert surfaces $S$ of genus $2 n+g$ which are mutually non-weakly equivalent. 
Proposition 6.7 is proved by showing that different surfaces are carried by an incompressible branched surface with a different set of weights $\left\{m_{1}, \ldots m_{n}\right\}$ so that $m_{1}+m_{2}+\ldots m_{n}=g$. Two surfaces are weakly equivalent if and only if $m_{i}=m_{i}^{\prime}$ for each $i=1, \ldots, n$.

Now let $L=l_{1} \cup l_{2}$ be a 2 -bridge link and $f: \partial E(L) \rightarrow \partial E\left(L^{n}\right)$ be an orientation reversing homeomorphism mapping a meridian of $l_{i}$ to a longitude of $l_{i}^{n}$. Define $M=E(L) \cup_{f} E\left(L^{n}\right)$. This gluing induces a strongly irreducible Heegaard splitting of $M$ of genus $4 n+2+2 g$. If $g>n$ these Heegaard splitting are not of minimal genus.

For the Heegaard splittings of the manifold $M$ we have:

Proposition 6.8 (Kobayashi [27]) If $L$ is not a $(2,2 n)$ torus link and two Heegaard splittings of $M$ induced by the Seifert surfaces $S$ and $S^{\prime}$ are homeomorphic then the homeomorphism is a weak equivalence between $S$ and $S^{\prime}$.

Note that in all the examples so far (Kobayashi [24, 27], Lustig-Moriah [40] and Moriah-Schultens [50]), the knots/links $K \subset S^{3}$ in question all have the property that the higher, non-minimal genus splittings are obtained by iteratively twisting the knot in a Conway sphere through a $\pi$ angle via an ambient isotopy of $S^{3}$. Though the knot type stays the same the new "position" of the knot induces a new Heegaard splitting which has genus bigger by two than the previous one.

One should also note that all the Heegaard splittings discussed above are strongly irreducible and two Heegaard splittings become isotopic after stabilizing once the higher genus and the corresponding number of stabilizations for the lower genus splitting.

A set of somewhat different examples were introduced by Moriah, Schleimer and Sedgwick in [49]. These examples are not related to knot spaces but they are brought here to emphasize the fact that the phenomenon of manifolds with strongly irreducible Heegaard splittings of arbitrarily high genus is very common.

Note that the manifolds of Casson-Gordon have Heegaard genus four and larger. These new examples have genus as low as three. Also, these examples, unlike those of [27] and [40], do not involve twisting around a two-sphere in $S^{3}$ or require the existence of an incompressible spanning surface.

Here is a sketch of the construction, which has obvious generalizations: Let $V$ be a handlebody of genus three or more. Choose $\gamma$ to be a "disk busting" curve in $S=\partial V$, that is, a curve which intersects every essential disk in $V$. Note that $K^{\prime}=S-N(\gamma)$ is an incompressible surface in $V$. Let $W$ be another copy of $V$ and now double $V$ across $S$ after modifying the gluing of $V$ to $W$ by Dehn twisting along $\gamma$ at least six times. 
This gives a closed orientable manifold $M$. As $K^{\prime} \cap \gamma=\emptyset$ the surface $K^{\prime}$ doubles to give a surface $K$ in $M$. which intersects $S$ transversally. Adding copies of $K$ to $S$ via the Haken sum operation (see [49]) will give the desired sequence of Heegaard splittings. This requires proving the following theorem (see [49]):

Theorem 6.9 Given $V$ and $\gamma$ as above, the surface $S+n K$ is a strongly irreducible Heegaard splitting of $M$, for any even $n>0$.

It is clear from the construction that the genus of the Heegaard surface $S+n K$ goes up as $n$ increases.

Remark 6.10 There are examples of complements of links in $S^{3}$ which have weakly reducible non-stabilized Heegaard splittings. The idea behind all of these examples is that each of these spaces has Heegaard splittings which have different partitions of the boundary components in the compression bodies. The first such example is due to Sedgwick [77] and is the three component unlink in $S^{3}$. It has a minimal genus two Heegaard splitting where one compression body contains two of the boundary components and the other compression body contains one. It also has a clearly weakly reducible non-stabilized genus three Heegaard splitting with all three boundary components in a single compression body. In further work by Moriah and Sedgwick [51] two infinite sets of different examples of two component links $L \subset S^{3}$ with genus three weakly reducible non-stabilized Heegaard splittings of $E(L)$ which are not minimal genus, were found.

So after all of the results and discussion above we have still made no progress regarding the nemesis of Heegaard splittings: Weakly reducible but irreducible Heegaard splittings?

\section{Additivity of Tunnel number}

Given two knots $K_{1}, K_{2} \subset S^{3}$ it is a natural question to ask: How does the genus of $E\left(K_{1}\right)$ and $E\left(K_{2}\right)$ compare with the genus of the connected sum $E(K)=E\left(K_{1} \# K_{2}\right)$ ? This problem is usually referred to as the additivity of tunnel number.

It is a well known fact that gluing two compression bodies $V_{1}$ and $V_{2}$ along an annulus $A$ will result in a compression body $V=V_{1} \cup_{A} V_{2}$, if and only if $A$ is primitive, as in Definition 5.13, in either $V_{1}$ or $V_{2}$. For a proof of this result and an extension see [47].

Recall that given two knots $K_{1}, K_{2} \subset S^{3}$ the exterior $E(K)$ of the connected sum $K=K_{1} \# K_{2}$ is obtained by gluing $E\left(K_{1}\right)$ to $E\left(K_{2}\right)$ along a meridional annulus $A$. 
As a consequence, it is a fact that for all knots $K=K_{1} \# K_{2} \subset S^{3}$ the tunnel numbers satisfy $t(K) \leq t\left(K_{1}\right)+t\left(K_{2}\right)+1$ : Let $\left\{\tau_{1}, \ldots, \tau_{t\left(K_{1}\right)}\right\}$ be a minimal tunnel system for $K_{1}$ and $\left\{\sigma_{1}, \ldots, \sigma_{t\left(K_{2}\right)}\right\}$ be a minimal tunnel system for $K_{2}$. Let $A$ denote the decomposing annulus for $K=K_{1} \# K_{2}$ and let $a$ be an essential arc on $A$. The arc $a$ is an unknotting tunnel for say $K_{1}$ (It can be thought of as an unknotting tunnel for $K_{2}$ as well.) As if it is slightly pushed into the handlebody $W_{1}=S^{3}-N\left(K_{1} \cup\left\{\cup \tau_{i}\right\}\right)$ in a neighborhood of $A$ it clearly has a dual disk in $W_{1}$. Furthermore $A$ becomes a primitive annulus in $W_{1}$ because its core curve intersects the same disk in a single point. Hence if $W_{2}$ is the handlebody complement of $S^{3}-N\left(K_{2} \cup\left\{\cup \sigma_{i}\right\}\right)$ then $W_{1} \cup_{A} W_{2}$ is a handlebody. So $\left\{a, \tau_{1}, \ldots, \tau_{t\left(K_{1}\right)}, \sigma_{1}, \ldots, \sigma_{t\left(K_{2}\right)}\right\}$ is a tunnel system of cardinality $t\left(K_{1}\right)+t\left(K_{2}\right)+1$ for $K$.

In other words the Heegaard splittings of $E\left(K_{1}\right)$ and $E\left(K_{2}\right)$ determined by

$$
\left\{a, \tau_{1}, \ldots, \tau_{t\left(K_{1}\right)}\right\} \quad \text { and } \quad\left\{\sigma_{1}, \ldots, \sigma_{t\left(K_{2}\right)}\right\}
$$

induce a Heegaard splitting, as in Definition 5.14, on $E(K)$.

Hence there are three possibilities for $K$ :

Super additive $t(K)=t\left(K_{1}\right)+t\left(K_{2}\right)+1$, that is,

$$
g(E(K))=g\left(E\left(K_{1}\right)\right)+g\left(E\left(K_{2}\right)\right) .
$$

Additive $t(K)=t\left(K_{1}\right)+t\left(K_{2}\right)$, that is,

$$
g(E(K))=g\left(E\left(K_{1}\right)\right)+g\left(E\left(K_{2}\right)\right)-1 .
$$

Sub-additive $t(K)<t\left(K_{1}\right)+t\left(K_{2}\right)$, that is,

$$
g(E(K)) \leq g\left(E\left(K_{1}\right)\right)+g\left(E\left(K_{2}\right)\right)-2 .
$$

Before we discuss the three cases we need some definitions and context.

Definition 7.1 Suppose $\mathcal{T} \subset V$ is a disjoint collection of properly embedded arcs in a compression body $V$ where $\partial \mathcal{T} \subset \partial_{+} V$. We say $\mathcal{T}$ is unknotted if $\mathcal{T}$ can be properly isotoped, rel boundary, into $\partial V$.

The following well-known generalization of bridge position is due to Doll [10]:

Definition 7.2 Suppose that $M=V \cup_{S} W$ and $K$ is a knot in $M$. The knot $K$ is in bridge position with respect to $S$ if $K$ is transverse to $S$ and either

(1) $K \cap S \neq \emptyset$, and both $K \cap V$ and $K \cap W$ are unknotted, or 
(2) $K \cap S=\emptyset, K \subset V$ (without loss of generality) and $V-N(K)$ is a compression body.

If $g=g(S)$ and $b=|K \cap S| / 2$ then we say that $K$ admits a $(g, b)$-decomposition. Saying that $K$ has no $(g, b)$-decompositions means that $K$ admits no $\left(g^{\prime}, b^{\prime}\right)$-decompositions with $g^{\prime} \leq g, b^{\prime} \leq b$.

There is a connection between the notions of additivity of tunnel number and $(g, b)-$ decompositions. It is exhibited, for example, in the following known fact:

Lemma 7.3 Let $K \subset M$ be a knot with $t(K)=t$. Then $K$ has a $(t, 1)$-decomposition if and only is $K$ is $\mu$-primitive.

We give a proof to illustrate the connection.

Proof Let $(V, W)$ be the Heegaard splitting of genus $t$ of $M$ which realizes the $(t, 1)$-decomposition. Hence $K=t_{1} \cup t_{2}$ where $t_{1} \subset V, t_{2} \subset W$ are unknotted arcs. Consider a regular neighborhood of $t_{2} \subset W$. We can think of it as a 1-handle containing $t_{2}$. Remove it from $W$ and add it to $V$ to obtain a handlebody $V^{\prime}$ of genus $t+1$. Since $t_{2}$ was unknotted the drilled out manifold $W^{\prime}$ is also a handlebody. The cocore disk of the 1-handle meets an essential disk of $W^{\prime}$ in a single point. This disk is the disk which is determined by the given isotopy of $t_{2}$ into $\partial_{+} W$. When $K \subset V^{\prime}$ is removed we get a compression body $V^{\prime \prime}$. Thus $\left(V^{\prime \prime}, W^{\prime}\right)$ is a Heegaard splitting of minimal genus $g=t+1$ and by the construction is $\mu$-primitive.

If $(V, W)$ is a minimal genus Heegaard splitting for $M-N(K)$ with $V$ being the compression body then add a solid torus neighborhood of $K$ to $V$ to obtain a handlebody $V^{\prime}$. It has an essential disk $D$ which meets $K$ in a single point. Since $(V, W)$ is $\mu$-primitive there is an essential disk $E$ of $W$ which $D$ meets in a single point. If we remove a regular neighborhood $N(D)$ of $D$ from $V^{\prime}$ and add it to $W$ along an annulus as a 2-handle we obtain handlebodies $V^{\prime \prime}$ and $W^{\prime \prime}$ and hence a Heegaard splitting $\left(V^{\prime \prime}, W^{\prime \prime}\right)$ of genus $g-1=t(K)$ for $M$. Now $K \cap N(D)$ is an $\operatorname{arc} t_{2} \subset W^{\prime \prime}$ and $K \cap V^{\prime \prime}$ is an $\operatorname{arc} t_{1}$ and they are clearly unknotted. So we have a $(t, 1)$-decomposition for $K \subset M$.

We will discuss the topic of additivity of tunnel number according to the above partition into super additive, additive and sub-additive. However this partition is somewhat artificial and hard to enforce as various results in one section are relevant and could be seen as belonging to another. 


\section{1 (1) Super Additive}

It clearly follows from the above discussion and Definitions 5.13 and 5.14 that if either of the knots $K_{i}, i=1,2$ is $\mu$-primitive then case (1), as in the trichotomy above, cannot happen.

Remark 7.4 For a long time it was an open question whether there are any knots that satisfy super additivity. This was finally resolved by Moriah-Rubinstein [48] and Morimoto, Sakuma and Yokota [58].

In [48] the following theorem was proved:

Theorem 7.5 (Moriah and Rubinstein [48]) For any pair of odd positive integers $r, s \geq 3$ there exist infinitely many pairs of knots $K_{1}, K_{2} \subset S^{3}$ so that $t\left(K_{1}\right)=(r-1) / 2$, $t\left(K_{2}\right)=(s-1) / 2$ and $t\left(K_{1} \# K_{2}\right)=t\left(K_{1}\right)+t\left(K_{2}\right)+1=(r+s) / 2$.

The above Theorem 7.5 is an existence theorem and is not constructive in the following sense: The knots $K_{1}$ and $K_{2}$ are obtained as the branched sets in $S^{3}$ of a 2-fold cover of $S^{3}$ by "sufficiently large " $m / n$-surgery on pretzel knots $K_{1}=K\left(p_{1}, \ldots, p_{r}\right)$ and $K_{2}=K\left(p_{1}^{\prime}, \ldots, p_{s}^{\prime}\right)$ where $r, s$ are odd, $p_{i}=p_{r-i+1}$, and $p_{i}^{\prime}=p_{s-i+1}^{\prime}$. As we cannot determine the precise number $m / n$ we can only get an existence result.

In [58] Morimoto, Sakuma and Yokota actually give the first concrete examples of knots which are super additive. These knots are all tunnel number one knots. They are of the following form:

A knot in $S^{3}$ will be called a twisted torus knot of the form $T(p, q, r, n) r<\max \{p, q\}$ if it is isotopic to a $(p, q)$-torus knot in which $r$ adjacent strands are twisted $n$ full twists. Consider the twist knot $K_{m}=T(7,17,2,5 m-3)$. The result then follows from the following two theorems recalling an old result by Norwood, see [61]. He proves that a knot a with a fundamental group that is generated by two elements is prime. Hence tunnel number one knots must be prime.

Theorem 7.6 (Morimoto, Sakuma and Yokota [58]) For all integers $m$ the knots $K_{m}$ do not have a $(1,1)$-decomposition.

For tunnel number one knots we have the following theorem by Morimoto [52]:

Theorem 7.7 (Morimoto [52]) Let $K_{1}$ and $K_{2}$ be tunnel number one knots. Then $K_{1} \# K_{2}$ has tunnel number two if and only if at least one of $K_{1}$ and $K_{2}$ admits a $(1,1)$-decomposition. 
More examples of tunnel number one knots which do not have $(1,1)$-decompositions have been discovered by Eudave-Muñoz in [11]. They are quite complicated so we will not describe them here. Recently Johnson and Thompson [22] have proved:

Theorem 7.8 (Johnson and Thompson [22]) For every $n \in \mathbb{N}$, there is a tunnel number one knot $K$ such that $K$ does not have a $(1, n)$ decomposition.

A stronger theorem which generalizes the above was also recently proved by Minsky, Moriah and Schleimer [43]:

Theorem 7.9 (Minsky, Moriah and Schleimer [43]) For any positive integers $t$ and $b$ there is a knot $K \subset S^{3}$ with tunnel number $t$ so that $K$ has no $(t, b)$-decomposition.

One should point out that both of the above theorems are not constructive.

The above theorem was proved utilizing the following theorem, which is of independent interest, and work of Scharlemann-Tomova [70] and Tomova which generalizes her work in [79]:

Theorem 7.10 For any pair of integers $g>1$ and $n>0$ there is a knot $K \subset S^{3}$ and a genus $g$ splitting of $E(K)$ having distance greater than $n$.

Hence all of the above knots $K$ which are tunnel number one knots must have $t(K \# K)=t(K)+t(K)+1=3$. The emergence of these examples in recent years led to the following conjecture by Morimoto [55] and separately by the author [47]:

Conjecture 7.11 If $K_{1}$ and $K_{2}$ are knots in $S^{3}$ then $t\left(K_{1} \# K_{2}\right)=t\left(K_{1}\right)+t\left(K_{2}\right)+1$ if and only if neither of $K_{1}$ and $K_{2}$ are $\mu$-primitive.

Remark 7.12 The conjecture is known to be true by work of Morimoto for tunnel number one knots [52] and for knots which are connected sums of two prime knots each of which is also $m$-small [55]. It has been further generalized by Kobayashi and Rieck [34] to general knots which are $m$-small, that is, knots which do not contained essential surfaces with meridional boundary components.

However the situation is not that simple as there is some evidence for the falsity of the conjecture:

Let $n K$ denote $K \# K \# \cdots \# K, n$ times. 
(1) It is a theorem of Kobayashi-Rieck [33] that if there exists a knot $K \subset S^{3}$ such that both $K$ and $2 K=K \# K$ are not $\mu$-primitive then the above Conjecture 7.11 is false.

(2) It is also a theorem of theirs [33] that if a knot $K$ has a $(t, n)$-decomposition then the knot $n K$ is $\mu$-primitive.

Note that all actual known examples of knots which are not $(t, 1)$ are $(t, 2)$. Hence they fail as candidates for a counter example to the above conjecture.

Note also that (1) follows from (2). Here is the proof given in [33]: Let $m$ be the minimal positive integer so that $t(m K)<m t(K)+(m-1)$. Such an $m$ exists because of (2) and $n \geq 2$. If $m=2$ we are done as $K$ is the counter example.

Assume that $m \geq 3$. Set $K_{1}=2 K$ and $K_{2}=(m-2) K$. By the assumption and the minimality of $m$ we have $t\left(K_{1}\right)=2 t(K)+1$ and $t\left(K_{2}\right)=(m-2) t(K)+(m-3)$. However $t\left(K_{1} \# K_{2}\right)=t(m K)<m t(K)+(m-1)=t\left(K_{1}\right)+t\left(K_{2}\right)+1$. So $K_{1}$ and $K_{2}$ are a contradiction to Conjecture 7.11.

We give a proof for (2) in Section 7.2 below. Hence they asked the following question [33, 1.9]: Are there knots $K \subset S^{3}$ so that $t(K)=t$ and which admit a $(t, n)$-decomposition with $n$ minimal and $n \geq 3$ ?

Remark 7.13 Theorems 7.9 and 7.8 give a positive answer to the above question. However showing that a knot $K$ which is a non-trivial connected sum has a $(t(K), b)-$ decomposition must be very tricky since these knots contain essential tori so all their Heegaard splittings are distance at most 2 by Hempel [17]. Hence the idea behind the proof of Theorems 7.9 and 7.8 will not work in this case and one needs a completely different technique.

Though the above Remark 7.13 shows that we are far away from finding a counterexample to the Morimoto Conjecture using these techniques it is the belief of the author that the conjecture is false. In fact, very recently the existence of counter examples has been announced by Kobayashi and Rieck [30, 31]. A better conjecture would be:

Conjecture 7.14 If $K_{1}, K_{2}$ are prime knots in $S^{3}$ then $t\left(K_{1} \# K_{2}\right)=t\left(K_{1}\right)+t\left(K_{2}\right)+1$ if and only if neither of $K_{1}$ and $K_{2}$ are $\mu$-primitive.

\section{2 (2) Additive}

If $K_{1} \subset S^{3}$ is $\mu$-primitive then for any other knot $K_{2} \subset S^{3}$ we have $t(K) \leq t\left(K_{1}\right)+t\left(K_{2}\right)$. What else can we say about knots which are $\mu$-primitive? The first thing to consider is knots which have more than one primitive meridian. 
Definition 7.15 We say that the knot $K \subset S^{3}$ is $n$-primitive if $E(K)$ has a minimal genus Heegaard splitting $(V, W)$ so that $\partial E(K) \subset V$ and with $n$ primitive meridians corresponding to $n$ disjoint pairwise non-isotopic disks $D_{1}, \ldots, D_{n} \subset W$.

Example 7.16 Let $K_{1}$ be a knot in a $2 n$-plat projection satisfying the following conditions:

Let $B$ denote the $2 n$-braid underlying the $2 n$-plat projection of $K$. Consider the image $\hat{\rho}(B)$ of $B$ under the Burau representation of the $2 n$-braid group, with the variable $t$ evaluated at -1 . Let $\alpha$ be the greatest common divisor of the entries of the matrix $L \circ \hat{\rho}(B) \circ M$. Assume that $\alpha \neq 1$. Let $\beta$ be the determinant of the matrix $N \circ \hat{\rho}(B) \circ M$. Then there is a minimal genus $n$ Heegaard splitting of $E(K)$ with $n$ different primitive meridians. In this case we have of course that $n=t(K)+1=g$. Furthermore if $K_{2}$ is another such knot then $t\left(K_{1} \# K_{2}\right)=t\left(K_{1}\right)+t\left(K_{2}\right)$ (see Lustig-Moriah [36]). In other words the set of knots which satisfy the conditions above is closed under connected sum.

It is clear that if $K$ has $n$ primitive meridians then this procedure can be iterated an arbitrary number of times with the corresponding inequality. That is, if $K_{1}, \ldots, K_{n} \subset S^{3}$ are any set of knots, then $t\left(K \# K_{1} \# \cdots \# K_{n}\right) \leq t(K)+t\left(K_{1}\right)+\cdots+t\left(K_{n}\right)$.

Definition 7.17 If $K \subset S^{3}$ such that $t(K)=t$ does not have a primitive meridian then it has a $(t, n)$ decomposition for some minimal $n$. We will say that $K$ has a $\frac{1}{n}$-primitive meridian.

In order to justify the above "multiplicative" language we prove the following theorem which is fact (2) of Remark 7.12. This proof is somewhat different than the one given in Kobayashi-Rieck [33].

Theorem 7.18 Suppose the knot $K$ has a $\frac{1}{n}$-primitive meridian; then $n K=K \# \cdots \# K$ $n$-times has a Heegaard splitting of genus $n t(K)+n$ which has a 1 -primitive meridian. That is, $n K$ is $\mu$-primitive.

Proof Let $K \subset S^{3}$ be a knot with a $(t(K), n)$ decomposition. Then there is a Heegaard splitting $\left(V_{1}, V_{2}\right)$ of $E(K)$ of genus $t(K)+n$ so that $\partial E(K) \subset V_{1}$. It is obtained by taking $n 1$-handles which are regular neighborhoods of the $n$ arcs $\left\{t_{1}, \ldots, t_{n}\right\}$ from one handlebody $W_{2}$ in the $(t(K), n)$ decomposition and adding them to the other handlebody $W_{1}$, then removing a smaller regular neighborhood of $K$ from the modified $W_{1}$ to obtain a compression body $V_{1}$ and a handlebody $V_{2}$ both of genus $t(K)+n$. 
The boundary of the cocore disks of the tunnels now determines a collection of $n$ simple closed curves $\left\{\gamma_{1}, \ldots, \gamma_{n}\right\} \subset \partial V_{2}$ which are by definition primitive. They have the additional property that the essential disks $\left\{D_{1}, \ldots, D_{n}\right\} \subset V_{2}$ that each curve $\left\{\gamma_{1}, \ldots, \gamma_{n}\right\} \subset \partial V_{2}$ intersect in a single point can be chosen to be pairwise disjoint and non-isotopic.

Now consider $\left(U_{1}^{1}, U_{2}^{1}\right), \ldots,\left(U_{1}^{n-1}, U_{2}^{n-1}\right), n-1$ copies of a minimal genus Heegaard splitting for $E(K)$ so that $\partial E(K) \subset U_{1}$. For each $i$ cut $U_{1}^{i}$ along a vertical annulus $A^{i}$ so that $\partial A^{i}=\alpha_{1} \cup \alpha_{2}$ with $\alpha_{1} \subset \partial E(K)$. This operation leaves two images $A_{1}^{i}, A_{2}^{i}$ of the annulus on the resulting handlebody. Similarly cut $V_{1}$ along $n-1$ vertical annuli $B_{i}$ corresponding to the cocore disks of the tunnels $\left\{t_{1}, \ldots, t_{n-1}\right\}$. Attach a copy of the cut open $U_{1}^{i}$ to the cut open $V_{1}$ by identifying the images of $A_{1}^{i}$ and $B_{1}^{i}$ and $A_{2}^{i}$ and $B_{2}^{i}$ to obtain a compression body.

Attach a copy of $U_{2}^{i}$ to $V_{2}$ by identifying the image of an annulus neighborhood of $\alpha_{2}$ in $\partial U_{2}^{i}$ with the annulus neighborhood of $\gamma_{i}$. Since $\gamma_{i}$ is primitive we obtain a handlebody of genus $t(K)+n+(n-1)(t(K)+1)-(n-1)=n t(K)+n$ which determines a Heegaard splitting $\left(H_{1}, H_{2}\right)$ of $E(n K)$. The meridian corresponding to the $t_{n}$ arc is clearly primitive from the construction meeting the essential disk $D_{n}$ of $H_{2}$ in a single point.

We now state some interesting facts:

Claim 7.19 If a knot $K \subset S^{3}$ is $\mu$-primitive then the corresponding Heegaard splitting $(V, W)$ has distance $d(V, W) \leq 2$.

Proof We assume that $\partial E(K) \subset V$. The compression body $V$ has many disks which are disjoint from the vertical annulus $A$ given by the assumption. Denote one such disk by $D_{0}$. Let $D \subset W$ be the disk intersecting $A$ in a single point. There is an essential disk $D^{\prime} \subset W$ composed of two copies of $D$ and a band running along the boundary component $a_{1}$ of $A$ contained in $\partial_{+} V$ which is disjoint from $a_{1}$. Hence $d_{\mathcal{C}(S)}\left(D_{0}, a_{1}\right)=1$ and $d_{\mathcal{C}(S)}\left(D^{\prime}, a_{1}\right)=1$ so the Heegaard splitting $(V, W)$ has distance $d(V, W) \leq 2$.

In [33] Kobayashi and Rieck make the following definition:

Definition 7.20 The growth rate of tunnel number is defined to be

$$
t_{\mathrm{gr}}(K)=\lim \frac{t(n K)-n t(K)}{n-1} .
$$

It is also referred to as the tunnel gradient. 
Proposition 7.21 If $K$ is a knot on a $2 n$-plat satisfying the conditions of Lustig and Moriah [36] then $t_{g r}(K)=0$.

Proof As $K$ satisfies the conditions of [36] the tunnel number is additive for connected sum of copies of $K$. It also has $n \geq 3$ primitive meridians and by Proposition 6.2 of [36] the set of knots which satisfy the [36] conditions is closed under connected sum. Hence we have $t(n K)=n t(K)$ so $t_{\mathrm{gr}}(K)=\lim \frac{t(n K)-n t(K)}{n-1}=0$.

Remark 7.22 Note that there are no known examples of knots $K \subset S^{3}$ so that $t(K \# K)<2 t(K)$. So we do not know of any knots with negative tunnel gradient $t_{\mathrm{gr}}(K)$.

\section{3 (3) Sub-additive}

The first result showing that there are knot exteriors which behave in a sub-additive way under connected sum is due to Morimoto in [54]. He proved the following (also mentioned in Remark 5.19):

Theorem 7.23 (Morimoto [54]) Let $K_{1}=K^{n}(-2,3,-3,2)$ be the twisted pretzel, where between the two 3-strands of twists one introduces an odd number $n \in \mathbb{Z}, n \notin$ $\{-1,0,1\}$ of "horizontal" crossings, and $K_{2}$ is any 2 -bridge knot. Then $t\left(K_{1}\right)=2$ and $t\left(K_{1} \# K_{2}\right)=2<2+1=t\left(K_{1}\right)+t\left(K_{2}\right)$.

This result was then generalized by Kobayashi [28] who proved:

Theorem 7.24 (Kobayashi [28]) For each positive integer $m$, there exist knots $K_{1}, K_{2}$ so that $t\left(K_{1}\right)+t\left(K_{2}\right)-t\left(K_{1} \# K_{2}\right)>m$.

Here is the idea of the proof:

The knot $K_{1}=2 m K^{n}$ is a connected sum of $2 m$ copies of $K^{n}$, the $n$ twisted pretzel $K^{n}(-2,3-3,2)$ as in Remark 5.19. The knot $K_{2}=6 m T(2,3)$ is a connected sum of $6 m$ copies of trefoils. It is known by [36] that $t\left(K_{2}\right)=6 \mathrm{~m}$ and Kobayashi proves, using the tangle structure of $K^{n}(-2,3-3,2)$, that $t\left(K_{1}\right) \geq 3 \mathrm{~m}$. Now, using the fact that connected sum is commutative, it follows from Morimoto's [54], above, that $t\left(K_{1} \# K_{2}\right) \leq 8 m$. Hence one obtains the required result that $t\left(K_{1}\right)+t\left(K_{2}\right)-t\left(K_{1} \# K_{2}\right)>m$.

Remark 7.25 The ideas in the above proof are put together craftily to achieve large degeneration of tunnel number; however, it is an iterated use of the Morimoto example [54]. There are no known other examples of two knots which are sub-additive. 
There are some results which give some conditions and bounds for the possible degeneration of tunnel number. The first ${ }^{3}$ such result was due to Morimoto and Schultens [59] who proved the following theorem:

Recall first that a knot $K \subset S^{3}$ is small if $E(K)$ does not contain a closed essential (incompressible and not boundary parallel ) surface. It follows from Culler, Gordon, Luecke and Shalen [9, Theorem 2.0.3] that if $E(K)$ contains an essential meridional (that is, a properly embedded bounded surface so that all boundary components are meridians of $K$ ) surface, then $E(K)$ contains a closed essential surface.

Theorem 7.26 (Morimoto-Schultens [59]) If both of $K_{1}, K_{2} \subset S^{3}$ are small then $t\left(K_{1}\right)+t\left(K_{2}\right) \leq t\left(K_{1} \# K_{2}\right)$.

That is, the presence of meridional essential surfaces is required for the degeneration of tunnel number. This result was later improved on by Morimoto [56] who proved:

Theorem 7.27 (Morimoto [56]) Let $M_{1}, \ldots, M_{n}$ be orientable closed 3-manifolds which do not have lens spaces summands. Let $K_{1} \subset M_{1}, \ldots, K_{n} \subset M_{n}$ be knots. If none of $E\left(K_{i}\right)=M_{i}-N\left(K_{i}\right), i=1, \ldots, n$ contain essential meridional surfaces then

$$
t\left(K_{1} \# \cdots \# K_{n}\right) \geq t\left(K_{1}\right)+\cdots+t\left(K_{n}\right) .
$$

In a slightly different flavor we have work of Scharlemann and Schultens:

Theorem 7.28 (Scharlemann-Schultens [67]) If $K_{i} \subset S^{3}, i=1, \ldots, n$, are nontrivial knots then $t\left(K_{1} \# \cdots \# K_{n}\right)>n$.

A much stronger result is:

Theorem 7.29 (Scharlemann-Schultens [68]) Let $K_{1}, \ldots, K_{n}$ be prime knots in $S^{3}$ then:

(1) $t\left(K_{1} \# \cdots \# K_{n}\right) \geq 1 / 3\left(t\left(K_{1}\right)+\cdots+t\left(K_{n}\right)\right)$.

(2) If none of the $K_{i}$ are 2-bridge knots then

$$
t\left(K_{1} \# \cdots \# K_{n}\right) \geq 2 / 5\left(t\left(K_{1}\right)+\cdots+t\left(K_{n}\right) .\right.
$$

(3) $t\left(K_{1} \# K_{2}\right) \geq 2 / 5\left(t\left(K_{1}\right)+t\left(K_{2}\right)\right)$.

\footnotetext{
${ }^{3}$ There is an earlier slightly weaker result of Schultens [73] which states: Theorem: For small knots $K_{1}, K_{2}, t\left(K_{1}\right)+t\left(K_{2}\right)-1 \leq t\left(K_{1} \# K_{2}\right) \leq t\left(K_{1}\right)+t\left(K_{2}\right)+1$.
} 
This leads to the following questions and conjecture:

Remark 7.30 We see from the above discussion that meridional essential surfaces are required for the tunnel number to degenerate. However it follows from Lustig-Moriah $[36,38]$ that there are many knots which contain many different meridional essential surfaces but for which the tunnel number is additive.

Hence:

Question 7.31 What are the properties of meridional essential surfaces which ensure that the tunnel number degenerate? Can these surfaces be classified?

Question 7.32 Are there knots which are not $K_{1}=K^{n}(-2,3,-3,2)$ and 2-bridge knots so that $t\left(K_{1} \# K_{2}\right)<t\left(K_{1}\right)+t\left(K_{2}\right)$ ?

Question 7.33 Are there knots which are not composites of $K_{1}=K^{n}(-2,3,-3,2)$ and 2-bridge knots so that $t\left(K_{1} \# K_{2}\right)<t\left(K_{1}\right)+t\left(K_{2}\right)-m$, for $m \geq 1$ ?

Conjecture 7.34 If $K_{1}$ and $K_{2}$ are prime knots for which $t\left(K_{1} \# K_{2}\right)<t\left(K_{1}\right)+t\left(K_{2}\right)$ then $t\left(K_{1} \# K_{2}\right)=t\left(K_{1}\right)+t\left(K_{2}\right)-1$.

\section{References}

[1] J Alexander, A lemma on systems of knotted curves, Proc. Nat. Acad. Sci. USA 9 (1963) 103-124

[2] D Bachman, D Cooper, ME White, Large embedded balls and Heegaard genus in negative curvature, Algebr. Geom. Topol. 4 (2004) 31-47 MR2031911

[3] S A Bleiler, Y Moriah, Heegaard splittings and branched coverings of $B^{3}$, Math. Ann. 281 (1988) 531-543 MR958258

[4] M Boileau, J-P Otal, Groupe des difféotopies de certaines variétés de Seifert, C. R. Acad. Sci. Paris Sér. I Math. 303 (1986) 19-22 MR849619

[5] M Boileau, M Rost, H Zieschang, On Heegaard decompositions of torus knot exteriors and related Seifert fibre spaces, Math. Ann. 279 (1988) 553-581 MR922434

[6] M Boileau, H Zieschang, Heegaard genus of closed orientable Seifert 3-manifolds, Invent. Math. 76 (1984) 455-468 MR746538

[7] G Burde, H Zieschang, Knots, de Gruyter Studies in Mathematics 5, Walter de Gruyter \& Co., Berlin (1985) MR808776 
[8] A J Casson, C M Gordon, Reducing Heegaard splittings, Topology Appl. 27 (1987) 275-283 MR918537

[9] M Culler, C M Gordon, J Luecke, P B Shalen, Dehn surgery on knots, Ann. of Math. (2) 125 (1987) 237-300 MR881270

[10] H Doll, A generalized bridge number for links in 3-manifolds, Math. Ann. 294 (1992) 701-717 MR1190452

[11] M Eudave-Muñoz, Incompressible surfaces and (1, 1)-knots, J. Knot Theory Ramifications 15 (2006) 935-948 MR2251034

[12] $\mathbf{R}$ H Fox, Free differential calculus I: Derivation in the free group ring, Ann. of Math. 55 (1953) 547-560

[13] K Funcke, Nicht frei äquivalente Darstellungen von Knotengruppen mit einer definierierenden Relation, Math. Z. 141 (1975) 205-217 MR0372044

[14] C M Gordon, J Luecke, Knots with unknotting number 1 and essential Conway spheres, Algebr. Geom. Topol. 6 (2006) 2051-2116 MR2263059

[15] C M Gordon, A W Reid, Tangle decompositions of tunnel number one knots and links, J. Knot Theory Ramifications 4 (1995) 389-409 MR1347361

[16] Y Hagiwara, Reidemeister-Singer distance for unknotting tunnels of a knot, Kobe J. Math. 11 (1994) 89-100 MR1309994

[17] J Hempel, 3-manifolds, Annals of Mathematics Studies 86, Princeton University Press (1976)

[18] J Hempel, 3-manifolds as viewed from the curve complex, Topology 40 (2001) 631-657 MR1838999

[19] W Jaco, Lectures on three-manifold topology, CBMS Regional Conference Series in Mathematics 43, American Mathematical Society, Providence, R.I. (1980) MR565450

[20] K Johannson, Heegaard surfaces in Haken 3-manifolds, Bull. Amer. Math. Soc. (N.S.) 23 (1990) 91-98 MR1027902

[21] K Johannson, Topology and combinatorics of 3-manifolds, Lecture Notes in Mathematics 1599, Springer, Berlin (1995) MR1439249

[22] J Johnson, A Thompson, On tunnel number one knots which are not $(1, n)$ arXiv: math.GT/0606226

[23] I Kapovich, R Weidmann, Kleinian groups and the rank problem, Geom. Topol. (to appear)

[24] T Kobayashi, There exist 3-manifolds with arbitrarily high genus irreducible Heegaard splittings, unpublished

[25] T Kobayashi, Structures of the Haken manifolds with Heegaard splittings of genus two, Osaka J. Math. 21 (1984) 437-455 MR752472

[26] T Kobayashi, A criterion for detecting inequivalent tunnels for a knot, Math. Proc. Cambridge Philos. Soc. 107 (1990) 483-491 MR1041480 
[27] T Kobayashi, A construction of 3-manifolds whose homeomorphism classes of Heegaard splittings have polynomial growth, Osaka J. Math. 29 (1992) 653-674 MR1192734

[28] T Kobayashi, A construction of arbitrarily high degeneration of tunnel numbers of knots under connected sum, J. Knot Theory Ramifications 3 (1994) 179-186 MR1279920

[29] T Kobayashi, Heegaard splittings of exteriors of two bridge knots, Geom. Topol. 5 (2001) 609-650 MR1857522

[30] T Kobayashi, Y Rieck, Knot exteriors with additive Heegaard genus and Morimoto's Conjecture arXiv:math.GT/0701765

[31] T Kobayashi, Y Rieck, Knots with $g(E(K))=2$ and $g(E(K \# K \# K))=6$ and Morimoto's Conjecture arXiv:math.GT/070176

[32] T Kobayashi, Y Rieck, Manifolds admitting both strongly irreducible and weakly reducible Heegard splittings, preprint

[33] T Kobayashi, Y Rieck, On the growth rate of tunnel number of knots arXiv: math.GT/0402025

[34] T Kobayashi, Y Rieck, Heegaard genus of the connected sum of m-small knots, Comm. Anal. Geom. (to appear) arXiv:math.GT/0503229

[35] M Lustig, Y Moriah, Nielsen equivalence in Fuchsian groups and Seifert fibered spaces, Topology 30 (1991) 191-204 MR1098913

[36] M Lustig, Y Moriah, Generalized Montesinos knots, tunnels and $\mathcal{N}$-torsion, Math. Ann. 295 (1993) 167-189 MR1198847

[37] M Lustig, Y Moriah, Generating systems of groups and Reidemeister-Whitehead torsion, J. Algebra 157 (1993) 170-198

[38] M Lustig, Y Moriah, On the complexity of the Heegaard structure of hyperbolic 3-manifolds, Math. Z. 226 (1997) 349-358 MR1483536

[39] M Lustig, Y Moriah, Closed incompressible surfaces in complements of wide knots and links, Topology Appl. 92 (1999) 1-13 MR1670164

[40] M Lustig, Y Moriah, 3-manifolds with irreducible Heegaard splittings of high genus, Topology 39 (2000) 589-618 MR1746911

[41] M Lustig, Y Moriah, A finiteness result for Heegaard splittings, Topology 43 (2004) 1165-1182 MR2079999

[42] R C Lyndon, P E Schupp, Combinatorial group theory, Ergebnisse der Mathematik und ihrer Grenzgebiete 89, Springer, Berlin (1977) MR0577064

[43] Y Minsky, Y Moriah, S Schleimer, Knots of high distance, preprint

[44] Y Moriah, Heegaard splittings and group presentations, $\mathrm{PhD}$ thesis, University of Texas at Austin (1986)

[45] Y Moriah, Heegaard splittings of Seifert fibered spaces, Invent. Math. 91 (1988) 465-481 MR928492 
[46] Y Moriah, On boundary primitive manifolds and a theorem of Casson-Gordon, Topology Appl. 125 (2002) 571-579 MR1935173

[47] Y Moriah, Connected sums of knots and weakly reducible Heegaard splittings, Topology Appl. 141 (2004) 1-20 MR2058679

[48] Y Moriah, H Rubinstein, Heegaard structures of negatively curved 3-manifolds, Comm. Anal. Geom. 5 (1997) 375-412 MR1487722

[49] Y Moriah, S Schleimer, E Sedgwick, Heegaard splittings of the form $H+n K$, Comm. Anal. Geom. 14 (2006) 215-247 MR2255010

[50] Y Moriah, J Schultens, Irreducible Heegaard splittings of Seifert fibered spaces are either vertical or horizontal, Topology 37 (1998) 1089-1112 MR1650355

[51] Y Moriah, E Sedgwick, Closed essential surfaces and weakly reducible Heegaard splittings in manifolds with boundary, J. Knot Theory Ramifications 13 (2004) 829-843 MR2088748

[52] K Morimoto, On the additivity of tunnel number of knots, Topology Appl. 53 (1993) 37-66 MR1243869

[53] K Morimoto, On composite tunnel number one links, Topology Appl. 59 (1994) 59-71 MR1293117

[54] K Morimoto, There are knots whose tunnel numbers go down under connected sum, Proc. Amer. Math. Soc. 123 (1995) 3527-3532 MR1317043

[55] K Morimoto, On the super additivity of tunnel number of knots, Math. Ann. 317 (2000) 489-508 MR1776114

[56] K Morimoto, Tunnel number, connected sum and meridional essential surfaces, Topology 39 (2000) 469-485 MR1746903

[57] K Morimoto, M Sakuma, On unknotting tunnels for knots, Math. Ann. 289 (1991) 143-167 MR1087243

[58] K Morimoto, M Sakuma, Y Yokota, Examples of tunnel number one knots which have the property “ $1+1=3$ ”, Math. Proc. Cambridge Philos. Soc. 119 (1996) 113-118 MR1356163

[59] K Morimoto, J Schultens, Tunnel numbers of small knots do not go down under connected sum, Proc. Amer. Math. Soc. 128 (2000) 269-278 MR1641065

[60] J Nielsen, Die Isomorphismen der allgemeinen, unendlichen Gruppe mit zwei Erzeugenden, Math. Ann. 78 (1964) 385-397 MR1511907

[61] F H Norwood, Every two-generator knot is prime, Proc. Amer. Math. Soc. 86 (1982) 143-147 MR663884

[62] P Ozsváth, Z Szabó, Knots with unknotting number one and Heegaard Floer homology, Topology 44 (2005) 705-745 MR2136532

[63] D Rolfsen, Knots and links, Mathematics Lecture Series 7, Publish or Perish, Berkeley, CA (1976) MR0515288 
[64] H Rubinstein, M Scharlemann, Comparing Heegaard splittings of non-Haken $3-$ manifolds, Topology 35 (1996) 1005-1026 MR1404921

[65] M Sakuma, Manifolds with infinitely many non-isotopic Heegaard splittings of minimal genus, from: "(Unofficial) Proceedings of the Conference on Various Structures on Knots and their Applications (Osaka City University)" (1988) 172-179

[66] M Scharlemann, There are no unexpected tunnel number one knots of genus one, Trans. Amer. Math. Soc. 356 (2004) 1385-1442 MR2034312

[67] M Scharlemann, J Schultens, The tunnel number of the sum of $n$ knots is at least $n$, Topology 38 (1999) 265-270 MR1660345

[68] M Scharlemann, J Schultens, Comparing Heegaard and JSJ structures of orientable 3-manifolds, Trans. Amer. Math. Soc. 353 (2001) 557-584 MR1804508

[69] M Scharlemann, A Thompson, Heegaard splittings of (surface) $\times I$ are standard, Math. Ann. 295 (1993) 549-564

[70] M Scharlemann, M Tomova, Alternate Heegaard genus bounds distance, Geom. Topol. 10 (2006) 593-617 MR2224466

[71] H Schubert, Knoten mit zwei Brücken, Math. Z. 65 (1956) 133-170

[72] J Schultens, The classification of Heegaard splittings for (compact orientable surface) $\times S^{1}$, Proc. London Math. Soc. (3) 67 (1993) 425-448 MR1226608

[73] J Schultens, Additivity of tunnel number for small knots, Comment. Math. Helv. 75 (2000) 353-367 MR1793793

[74] J Schultens, R Weidmann, On the geometric and algebraic rank of graph manifolds, preprint

[75] E Sedgwick, An infinite collection of Heegaard splittings that are equivalent after one stabilization, Math. Ann. 308 (1997) 65-72 MR1446199

[76] E Sedgwick, The irreducibility of Heegaard splittings of Seifert fibered spaces, Pacific J. Math. 190 (1999) 173-199 MR1722770

[77] E Sedgwick, Genus two 3-manifolds are built from handle number one pieces, Algebr. Geom. Topol. 1 (2001) 763-790

[78] J Souto, A note on the tameness of hyperbolic 3-manifolds, Topology 44 (2005) 459-474 MR2114957

[79] M Tomova, Multiple bridge surfaces restrict knot distance arXiv:math.GT/0511139

Department of Mathematics, Technion

Haifa 32000, Israel

ymoriah@tx.technion.ac.il

Received: 4 August 2006 Revised: 30 March 2007 